\title{
STATE SPACES OF JORDAN ALGEBRAS
}

BY

ERIK M. ALFSEN and FREDERIC W. SHULTZ

University of Oslo

Oslo, Norway

Wellesley College

Wellesley, Massachussetts, USA

\section{Contents}

\$ 1. Introduction . . . . . . . . . . . . . . . . . . . . . . . . . . . . . . . . 155

\$ 2. Preliminaries and notation . . . . . . . . . . . . . . . . . . . . . . 157

§ 3. The Hilbert ball property . . . . . . . . . . . . . . . . . . . . . . . . . . 160

\$. The pure state properties. . . . . . . . . . . . . . . . . . . . . . . . . . . 168

\$ 5. The splitting into atomic and non-atomic parts . . . . . . . . . . . . . . . . . 171

§ 6. The type I factor case . . . . . . . . . . . . . . . . . . . . . . . . . . . . 175

\$7. The main theorem. . . . . . . . . . . . . . . . . . . . . . . . . . . 186

\section{\$1. Introduction}

The purpose of this paper is to give a geometric characterization of the state spaces of the class of normed Jordan algebras named $J B$-algebras in [4]. Recall in this connection that by the generalized Gelfand-Neumark theorem of [4] the study of $J B$-algebras can be reduced to the study of Jordan algebras of self-adjoint operators on a Hilbert space and the exceptional algebra $M_{3}^{8}$. One of the most important examples of a $J B$-algebra is the self-adjoint part of a $C^{*}$-algebra; thus the properties we establish for state spaces of $J B$-algebras also give information about state spaces of $C^{*}$-algebras.

We first prove that the state space of a $J B$-algebra has the following property which we term the "Hilbert ball property": for each pair $\varrho, \sigma$ of extreme points, the face generated by $\varrho$ and $\sigma$ is a norm-exposed face affinely isomorphic to the closed unit ball in some finite or infinite dimensional Hilbert space (Corollary 3.12). This observation appears to be new even for the case of a $C^{*}$-algebra.

We also prove that the $\sigma$-convex hull of the pure states of a $J B$-algebra is a split face of the state space (Corollary 5.8). In other words, the state space is a direct convex sum of two faces, one being the $\sigma$-convex hull of the extreme points, the other containing 11 - 772908 Acta mathematica 140. Imprimé le 9 Juin 1978 
no extreme points. This property, which we term the "splitting into atomic and nonatomic parts", follows from well known facts in the case of a $C^{*}$-algebra.

It is known that the state space of a $J B$-algebra is a strongly spectral compact convex set in the terminology of [2]. (Cf. [2; § 12] and [4; §4]). In the present paper we show that this property together with the two properties above characterize the state spaces of $J B$-algebras among all compact convex sets (Corollary 7.4).

In fact, we shall derive this result from a stronger theorem which does not invoke the full strength of the spectral theory of [2]. In order to state this theorem, we need the fundamental concept of a "projective face" of a convex set, defined in $[2 ; \S 2]$. (See also the geometric characterization of projective faces given in [2; Th. 3.8].) To fix the ideas, we note at this point that in the case of a $C^{*}$-algebra then the projective faces are in 1-1 correspondence with the self-adjoint projections (and the ultraweakly closed one-sided ideals) in the enveloping von Neumann algebra. We also recall that the projective faces occur in "quasicomplementary pairs" $F, F^{*}$ (corresponding to orthogonal projections $p, 1-p$ in the special case mentioned above). Moreover, two positive elements $a, b$ of the space $A$ of all bounded affined functions on $K$ are said to be "orthogonal", in symbols $a \perp b$, if there exists a projective face $F$ of $K$ such that $a=0$ on $F$ and $b=0$ on $F^{*}$. As usual, the space of continuous affine functions on $K$ is denoted by $A(K)$. Then we have the following:

MaIn Theorem. A compact convex set $K$ is the state space of a JB-algebra iff the following requirements are satisfied:

(i) $K$ has the Hilbert ball property

(ii) $K$ splits into an atomic and a non-atomic part

(iii) Every norm-exposed face of $K$ is projective

(iv) Every $a \in A(K)$ can be decomposed as a difference $a=b-c$ where $b, c \in A(K)^{+}$and $b \perp c$

(To say that $K$ is "strongly spectral" means that $K$ satisfies (iii) and (iv) above and in addition that every $a \in A$ can be decomposed as $a=b-c$ where $b, c \in A^{+}$and $b \perp c$, this decomposition being unique, ef. $[3 ; \S 2]$. For general compact convex sets, this additional requirement is irredundant, as shown by an example in $[3 ; \S 2]$.)

In the development leading up to the main theorem, we also establish another characterization of state spaces of $J B$-algebras. This characterization is less geometric in nature, but it is stated in terms of conditions which admit some interesting physical interpretations. Specifically, they are the "pure state properties" (4.3), (4.4) and (4.5) of $\S 4$, which are equivalent to physical axioms used in axiomatic quantum mechanics by various 
authors. (See $\S 4$ for further discussion.) Thus, our results establish further the connections between Jordan algebras, spectral theory and quantum mechanics, ef. [7], [9], [13], [16].

\section{§ 2. Preliminaries and notation}

Following [4] we define a JB-algebra to be a Jordan algebra over the reals with identity $e$ equipped with a complete norm such that for any two elements

$$
\begin{gathered}
\|a \circ b\| \leqslant\|a\|\|b\| \\
\left\|a^{2}\right\|=\|a\|^{2} \\
\left\|a^{2}\right\| \leqslant\left\|a^{2}+b^{2}\right\|
\end{gathered}
$$

A $J B$-algebra which is a Banach dual space, will be called a $J B W$-algebra.

The motivating example for $J B$ - and $J B W$-algebras are respectively the self adjoint part of a $C^{*}$-algebra and a von Neumann algebra, with the Jordan product $a \circ b=\frac{1}{2}(a b+b a)$. More generally, any Jordan operator algebra (norm closed Jordan algebra of self-adjoint operators on a Hilbert space with identity and the above product) is a $J B$-algebra.

The main result of [4] is that for every $J B$-algebra $B$ there is a unique ideal $J$ such that $B / J$ is (isometrically isomorphic to) a Jordan operator algebra and $J$ is exceptional in the sense specified in [4; Th. 9.5]. For $J B W$-algebras one has a stronger theorem, by which every $J B W$-algebra $A$ can be uniquely decomposed as a direct sum $A=A_{\mathrm{sp}} \oplus A_{\text {exc }}$ where $A_{\mathrm{sp}}$ is a Jordan operator algebra and $A_{\text {exc }}$ is the algebra $C\left(X, M_{3}^{8}\right)$ of all continuous functions from a hyperstonean space $X$ into $M_{3}^{8}$ [17]. ( $M_{3}^{8}$ is the "exceptional" Jordan algebra of self-adjoint $3 \times 3$-matrices over the Cayley numbers.)

In $[4 ; \S 3]$ it is shown that to every $J B$-algebra $B$ one can associate a "monotone complete enveloping $J B$-algebra" $\tilde{B}$. In [17] it is proved that $\tilde{B}$ can be identified with the bidual $B^{* *}$ equipped with the Arens product. In this paper we will refer to $\tilde{B}=B^{* *}$ as the enveloping $J B W$-algebra of $B$.

If $B$ is a $J B$-algebra, then we can order $B$ by the cone $B^{2}$ of all squares in $B$. With this ordering and the identity as order-unit, $B$ becomes an order-unit space. A state on $B$ is a positive linear functional with value 1 on the identity $e$. The state space $K$ of $B$ is the $w^{*}$-compact convex set of all states. For the dual ordering and norm, $B^{*}$ is a base-norm space with base $K$. (For definitions of order-unit and base-norm spaces see [1; Ch. II, §2].)

As shown in [17], the predual of a $J B W$-algebra is unique, and will be a base-norm space. The base of the positive cone in the predual can be identified with the set of normal states, i.e. the states $\varrho$ such that $\langle a, \varrho\rangle=\lim _{\alpha}\left\langle a_{\alpha}, \varrho\right\rangle$ whenever $\left\{a_{\alpha}\right\}$ is an increasing 
net with l.u.b. $a$. If $B$ is a $J B$-algebra with state space $K$, then $K$ can be identified with the normal state space of the enveloping $J B W$-algebra $B^{* *}$.

A $J B W$-algebra and its predual are in spectral duality by [3; Th. 3.1]; the state space of a $J B$-algebra is strongly spectral by [3; Cor. 3.2]. We will briefly review the basic notions of spectral theory from [2] and [3].

Let $(A, e)$ be an order-unit space which is the dual of a base-norm space $(V, K)$. Then one can identify $A$ with the space $A^{b}(K)$ of all bounded affine functions on $K$. We say two positive projections $P, Q$ on $A$ (or on $V$ ) are quasicomplementary (q.c.) if

$$
\operatorname{ker}^{+} P=\mathrm{im}^{+} Q, \quad \operatorname{im}^{+} P=\operatorname{ker}^{+} \mathbf{Q} .
$$

A $w^{*}$-continuous positive projection $P: A \rightarrow A$ with $\|P\| \leqslant 1$ is said to be a $P$-projection if there exists a (necessarily unique) $w^{*}$-continuous positive projection $P^{\prime}: A \rightarrow A$ with $\left\|P^{\prime}\right\| \leqslant 1$ such that $P, P^{\prime}$ are q.c. and the dual projections $P^{*}, P^{\prime *}$ (on $V$ ) are also q.c. (Various equivalent definitions are given in $[2 ; \S \S 2,3]$.) If $A$ is a $J B W$-algebra and $V$ its predual, then the $P$-projections are exactly the maps $U_{p}: a \mapsto\{p a p\}$ where $p$ is an idempotent and $\{$ pap $\}$ denotes the Jordan triple product. In particular $U_{p} a=p a p$ with $p$ a selfadjoint projection if $A$ is the self-adjoint part of a von Neumann algebra.

To every $P$-projection $P$ is associated a projective unit $u_{P}=P e$ and a projective face

$$
F_{P}=K \cap \operatorname{im} P^{*}=\{\varrho \in K \mid\langle P e, \varrho\rangle=1\} .
$$

The sets of $P$-projections on $A$, projective units in $A$, and projective faces of $K$ are denoted by $\mathcal{D}, \mathcal{U}$, and $\mathcal{F}$, respectively. In $\mathcal{D}$ an ordering is defined by $P \lessgtr Q$ when $P Q=Q P=P$; this is equivalent to the natural ordering of the corresponding elements of $\mathcal{U}$ and $\mathcal{F}$, i.e. to $P e \leqslant Q e$ and $F_{P} \subseteq F_{Q}$ (ef. [2; Lemma 2.16]). Thus the sets $\mathcal{U}, \mathcal{D}, \mathcal{F}$ are order isomorphic under the maps $P e \leftrightarrow P \leftrightarrow F_{P}$ [2; Th. 2.17]. Using these maps one can transfer the notion of a quasicomplement from $\mathcal{D}$ to $\mathcal{U}$ and $\mathcal{F}$. Specifically, let $u \in \mathcal{U}$ and $F \in \mathcal{F}$, say $u=P e$ and $F=F_{P}$ with $P \in \mathcal{D}$; then $u^{\prime}=P^{\prime} e=e-u$ and $F^{*}=F_{P^{\prime}}=K \cap \mathrm{im} P^{\prime *}$.

According to $[3 ; \S 2],(A, e)$ and $(V, K)$ are in spectral duality if the following two requirements are satisfied:

(2.5) Every norm-exposed face of $K$ is projective.

(2.6) Every $a \in A$ admits a unique decomposition $a=a^{+}-a^{-}$such that $a^{+}, a^{-} \in A^{+}$and $a^{+} \perp a^{-}$.

(Recall that a face $F$ of $K$ is norm-exposed if there exists a norm-closed supporting hyperplane which meets $K$ exactly in $F$; the orthogonality relation of (2.6) was defined in the introduction.) 
It is shown in [2] and [3] that if $(A, e)$ and $(V, K)$ are in spectral duality, then the set $\mathcal{F}$ of projective faces of $K$ is a complete orthomodular lattice (this depends only on (2.5)) and every $a \in A$ admits a unique spectral resolution. Furthermore, $A$ will possess a functional calculus for bounded Borel functions having the usual properties. This spectral resolution and functional calculus will coincide with those developed in $[4 ; \S 4]$ when $A$ is a $J B W$-algebra (cf. $[3 ; \S 2])$.

If in addition $K$ is compact in some locally convex Hausdorff topology, then it is of interest to know when the space $A(K)$ of continuous affined functions on $K$ will be closed under the functional calculus by continuous functions. By [2; Th. 10.6] and [3; §2] this will be true iff $K$ is a strongly spectral compact convex set, i.e. if $K$ satisfies (2.5) and (2.6) and also

$$
a^{+}, a^{-} \in A(K) \text { for all } a \in A(K)
$$

If $K$ is the state space of a $J B$-algebra $B$, then $B$ can be identified with $A(K)$ (up to order and norm isomorphism). Then $K$ will be strongly spectral, and the functional calculus from spectral theory will coincide with that in [4]; in particular the squaring maps will agree. Thus the Jordan product can be recovered from spectral theory by the formula $a \circ b=\frac{1}{2}\left((a+b)^{2}-a^{2}-b^{2}\right)$.

Assume now that $(A, e)$ is an order-unit space which is the dual of a base-norm space $(V, K)$ and that $(2.5)$ holds. We recall from $[2 ; \S 5]$ that a $P$-projection $P$ is central if $P+P^{\prime}=I$; by [2; Th. 10.2] this is equivalent to the corresponding projective face being a split face. We will say $A$ is a factor (for the given duality with $V$ ) if it contains no central $P$-projections other than 0 and $I$ (the identity map), or equivalently, if $K$ contains no proper split faces. We will say that $u \in \mathcal{U}$ is an atom if $u$ is a minimal (non-zero) projective unit, and we will say a factor $A$ is of type $I$ if it contains an atom. It follows from [3; Prop. 1.13] that if $u$ is an atom, then the corresponding projective face will contain exactly one (extreme) point, which we will henceforth denote by $\hat{u}$. Now $u \mapsto \hat{u}$ is a $1-1$ mapping of the atoms onto the norm-exposed points of $K$, and for a given atom $u$ then $\hat{u}$ is the unique point in $K$ such that $\langle u, \hat{u}\rangle=1$.

Specializing to a $J B W$-algebra $A$ (in duality with its predual), we note that an idempotent $p$ is central in the sense defined in [4] iff the corresponding $P$-projection $U_{p}$ is central in the above sense (cf. [4; Lemma 2.11]). A $J B W$-algebra $A$ which is a factor, will be called a $J B W$-factor. (The definition in $[4 ; \S 5]$ is slightly different, but via spectral theory and the results of [17] it can be seen to be equivalent.) Clearly, these notions for $J B W$-algebras will in turn specialize to the usual ones for von Neumann algebras. 


\section{§ 3. The Hilbert ball property}

We begin by recalling some general notions relating to an arbitrary convex set $K$ (in some linear space), and for brevity we denote by $A$ the space $A^{b}(K)$ of all bounded affine functions on $K$. (This conforms with the notations used in $\$ \S 1,2$.) For any subset $E$ of $K$ there exists a smallest face $F=$ face $(E)$ which contains $E$ (possibly $F=K$ ). We say that two extreme points $\varrho, \sigma$ of $K$ are separated by a split face if there exists a split face $F$ such that $\varrho \in F$ and $\sigma \in F^{\prime}$. (As usual $F^{\prime}$ denotes the complementary split face, cf. [1; Ch. II, §6].)

Proposition 3.1. If two extreme points $\varrho, \sigma$ of a convex set $K$ can be separated by $a$ split face, then face $(\{\varrho, \sigma\})$ is equal to the line segment $[\varrho, \sigma]$. If in addition $\varrho$ and $\sigma$ are normexposed points, then $[\varrho, \sigma]$ is a norm-exposed face.

Proof. 1. The statement that face $(\{\varrho, \sigma\})=[\varrho, \sigma]$, follows in a straightforward way from the definitions; we leave the details to the reader.

2. Assume now that $\varrho$ and $\sigma$ are norm-exposed, and let $a, b \in A$ be chosen such that $a>0$ on $K \backslash\{\varrho\},\langle a, \varrho\rangle=0$ and $b>0$ on $K \backslash\{\sigma\},\langle b, \sigma\rangle=0$. Also let $F$ be a split face such that $\varrho \in F$ and $\sigma \in F^{\prime}$. For every $\omega \in K$, let $\lambda(\omega) \in[0,1], \varphi(\omega) \in F^{\prime}$ and $\psi(\omega) \in F^{\prime}$ be (uniquely) determined by

$$
\omega=\lambda(\omega) \varphi(\omega)+(1-\lambda(\omega)) \psi(\omega)
$$

Now define a function $c$ on $K$ by writing

$$
\langle c, \omega\rangle=\lambda(\omega)\langle a, \varphi(\omega)\rangle+(1-\lambda(\omega))\langle b, \psi(\omega)\rangle
$$

Clearly $c$ is bounded, and it is seen by a straightforward verification that $c$ is affine. Hence $c \in A$. Clearly also $c>0$ on $K \backslash[\varrho, \sigma]$ and $c=0$ on $[\varrho, \sigma]$. This completes the proof.

By a Hilbert ball we shall understand the closed unit ball of some real Hilbert space $H$. The dimension of $H$ can be finite or infinite, and for convenience we also admit the "zero dimensional Hilbert ball", consisting of a single point.

Definition. A convex set $K$ has the Hilbert ball property if face $(\{\varrho, \sigma\})$ is a normexposed face affinely isomorphic to a Hilbert ball for every pair $\varrho, \sigma$ of extreme points of $K$.

Note that if the two extreme points $\varrho, \sigma$ coincide, then face $(\{\varrho, \sigma\})=$ face $\{\varrho\}$; so if $K$ has the Hilbert ball property, then every extreme point of $K$ is norm-exposed.

Note also that by Proposition 3.1, a convex set $K$ will have the Hilbert ball property iff every extreme point is norm-exposed and face $(\{\varrho, \sigma\})$ is a norm-exposed face affinely 
isomorphic to a Hilbert ball for every pair $\varrho, \sigma$ of distinct extreme points which can not be separated by a split face.

If $(A, e)$ is an order-unit space which is the dual of a base-norm space $(V, K)$ and if (2.5) holds, then every subset $E$ of $K$ is contained in a smallest projective (and normexposed) face [3; Cor. 1.2], which we will denote by $F(E)$. In this case, if the convex set $K$ has the Hilbert ball property, then

$$
F(\{\varrho, \sigma\})=\text { face }(\{\varrho, \sigma\})
$$

for every pair $\varrho, \sigma$ of extreme points of $K$; in particular

$$
F(\varrho)=\{\varrho\}
$$

for every single extreme point $\varrho$ of $K$.

We now proceed to prove that the normal state space of a $J B W$-algebra has the Hilbert ball property, and the first step will be to prove that every extreme point of the normal state space of a $J B W$-algebra $A$ is norm-exposed. We begin by showing this for the exceptional $J B$-algebra $M_{3}^{8}$ of all self-adjoint $3 \times 3$-matrices over the Cayley numbers. This algebra is finite dimensional, so we do not have to worry about normality for states, and we can use the term "exposed point" without further specification.

LEMMA 3.2. Every extreme point of the state space $K$ of $M_{3}^{8}$ is exposed.

Proof. Let $A=M_{3}^{8}$ and $V=\left(M_{3}^{8}\right)^{*}$. By a known result there exists in $A$ an inner product making $A^{+}$a self-dual cone, i.e. $(a \mid b) \geqslant 0$ for all $b \in A^{+}$iff $a \in A^{+}$(cf. [6; Ch. 11, Satz 3.8]). Now $a \mapsto(\cdot \mid a)$ is seen to be an order isomorphism of $A$ onto $A^{*}=V$. Therefore, if we can prove that every extreme ray of $A^{+}$is exposed, then every extreme ray of $V^{+}$ will be exposed, and so every extreme point of $K$ will be exposed.

But an extreme ray of $A^{+}$is a closed face of $A^{+}$, and therefore of the form $\mathrm{im}^{+} P$ for some $P$-projection $P$ [2; Th. 12.3]. By Proposition 1.4 of [2], im $+P$ is semi-exposed, and by finite dimensionality, exposed. This completes the proof.

Proposition 3.3. Every extreme point of the normal state space $K$ of a $J B W$-algebra $A$ is norm-exposed.

Proof. Let $\varrho$ be an extreme point of $K$ and let $c(\varrho)$ be the smallest central idempotent of $A$ satisfying $\langle c(\varrho), \varrho\rangle=1$. Also let $P_{\varrho}$ be the corresponding $P$-projection, i.e. $c(\varrho)=P_{\varrho} e$, and let $F_{Q}$ be the corresponding projective face, i.e. $F_{\varrho}=\{\sigma \in K \mid\langle c(\varrho), \sigma\rangle=1\}$. Note that $P_{\varrho}=U_{\text {o( } \varrho)}$. To show that $\varrho$ is norm-exposed, we will relativize to the spectral duality of $\left(A_{\varrho}, c(\varrho)\right)$ and $\left(V_{\varrho}, F_{\varrho}\right)$ where $A_{\varrho}=\operatorname{im} P_{\varrho}$ and $V_{\varrho}=\operatorname{im} P_{\varrho}^{*}$ (cf. [3; Prop. 2.3]). Specifically, we will show that $\{\varrho\}$ is a norm-exposed, or equivalently a projective, face of $F_{\varrho}$. 
By $\left[4 ;\right.$ Lemma 5.5.] $A_{\varrho}$ is a factor. Therefore by [4; Th. 8.6] $A_{\varrho}$ is either isomorphic to $M_{3}^{8}$ or to a Jordan operator algebra. In the former case we are done by Lemma 3.2. In the latter case the argument in the proof of Theorem 8.7 in [4] shows that the projective face generated by $\varrho$ is minimal. It then follows from [3; Prop. 1.13] that this face consists of $\varrho$ only. Thus, $\{\varrho\}$ is a projective face of $F_{\varrho}$, as desired.

Next we shall prove a series of lemmas needed for the study of face $(\{\varrho, \sigma\})$ for two extreme points $\varrho, \sigma$ of the normal state space of a $J B W$-algebra.

Lemma 3.4. Let $A$ be a $J B W$-algebra, and let $u, v$ be arbitrary idempotents in $A$. Then there exists $s \in A$ with $s^{2}=e$ such that

$$
U_{s}(u \vee v-u)=v-u \wedge v .
$$

Proof. By Lemma 6.2 of [4] there exists $s \in A$ with $s^{2}=e$ such that

$$
U_{s}\left\{u^{\prime} v u^{\prime}\right\}=\left\{v u^{\prime} v\right\}
$$

where $u^{\prime}=e-u$. By the proof of Lemma 6.3 of [4] we also have

$$
U_{s} r\left(\left\{u^{\prime} v u^{\prime}\right\}\right)=r\left(\left\{v u^{\prime} v\right\}\right) .
$$

By Lemma 1.8 and equation (1.4) of [3]:

and

$$
r\left(\left\{u^{\prime} v u^{\prime}\right\}\right)=(v \vee u) \wedge u^{\prime}=(v \vee u)-u
$$

which completes the proof.

The following corollary will not be needed in the sequel, but seems of interest in its own right. Let $L$ be a lattice and $a, b \in L$; one says $(a, b)$ is a modular pair (written $(a, b) M$ ) if for all $x \in L$ with $a \wedge b \leqslant x \leqslant b$ there holds $x=(x \vee a) \wedge b$. A lattice is semimodular if the relation $M$ is symmetric, i.e. if $(a, b) M$ implies $(b, a) M$. (For background see [5] or [11].) Topping [20] has shown that the projection lattice of a von Neumann algebra is semimodular. We will now generalize this to $J B W$-algebras.

CoR OLLARY 3.5. The lattice $U$ of idempotents (=projective units) in a $J B W$-algebra $A$ is semimodular.

Proof. We know that $U$ is an orthomodular lattice (cf. [2; Th. 4.5] or [4; Prop. 4.9]). By Corollary 36.14 and Theorem 29.8 of [11], it now suffices to prove that whenever $u, v \in \mathcal{U}$ and $u \vee v=e, u \wedge v=0$, there exists a lattice automorphism of $u$ taking $u$ to $v^{\prime}$ and $v$ to $u^{\prime}$. 
If $s \in A$ and $s^{2}=e$, then the map $U_{s}: a \mapsto\{s a s\}$ satisfies $U_{s}^{2}=I$. Note that since $U_{s}$ is a Jordan automorphism (cf. [8; p. 60] or use Macdonald's Theorem [8; p. 4l]), then it maps idempotents into idempotents; by [4; Prop. 2.7] it is order preserving so it is a lattice automorphism of $\mathcal{U}$.

Now if $u \vee v=e$ and $u \wedge v=0$, then Lemma 3.4 gives $U_{s}(e-u)=v$ for some $s \in A$ with $s^{2}=e$. Thus $U_{s} u=e-v=v^{\prime}$ and $U_{s} v=u^{\prime}$, which completes the proof that $\mathcal{U}$ is semimodular.

L⿴囗十м 3.6. If $u$ is a minimal idempotent of a JBW-algebra and $v$ is an arbitrary idempotent, then $u \vee v-v$ is either a minimal idempotent or zero.

Proof. By Lemma 3.4 there exists $s \in A$ with $s^{2}=e$, such that $U_{s}$ exchanges $u \vee v-v$ and $v-u \wedge v$. By minimality of $v$, the latter either equals $v$ or is zero. Now $U_{s}$ maps minimal idempotents to minimal idempotents. This proves the lemma.

LEMMA 3.7. Let $u, v$ be distinct minimal idempotents of a $J B W$-algebra. If $w$ is an idempotent different from 0 and $u \vee v$ such that $w<u \vee v$, then $w$ is also minimal.

Proof. Define $w^{*}=u \vee v-w$. Then $u \leqslant u \vee w^{*} \leqslant u \vee v$; we claim one of these relations is actually an equality. For if both inequalities are strict, then we also have the strict inequalities

$$
0<u \vee w^{*}-u<u \vee v-u
$$

which contradicts the fact that the last term is a minimal idempotent by Lemma 3.6. Thus, either $u=u \vee w^{*}$ or $u \vee w^{*}=u \vee v$.

In the former case, minimality of $u$ forces $u=w^{\sharp}$; so

$$
w=u \vee v-w^{*}=u \vee v-u
$$

which is minimal by Lemma 3.6.

In the latter case

$$
w=u \vee v-w^{*}=u \vee w^{*}-w^{*},
$$

which is again minimal by Lemma 3.6.

LEMMA 3.8. If $A$ is a JBW-factor and $u$ is a non-zero idempotent in $A$, then $\{u A u\}$ is a JBW-factor.

Proof. Note first that $\{u A u\}$ is a $J B W$-algebra, as can be seen from [4; Prop. 4.11].

Now suppose that $a$ is a non-trivial idempotent which is central in $\{u A u\}$, and let $b=u-a$. By Lemmas 6.4 and 6.7 of [4] there exist non-zero idempotents $a_{1} \leqslant a, b_{1} \leqslant b$ and 
$s \in A$ with $s^{2}=e$ such that $\left\{s a_{1} s\right\}=b_{1}$. Let $t=\{u s u\} ;$ then since $a_{1}, b_{1} \in\{u A u\}$

$$
\left\{t a_{1} t\right\}=\left\{u\left\{s\left\{u a_{1} u\right\} s\right\} u\right\}=b_{1} .
$$

But by positivity of the maps $U_{t}$ and $U_{b}$ [4; Prop. 2.7] we now get

$$
b_{1}=\left\{b b_{1} b\right\}=\left\{b\left\{t a_{1} t\right\} b\right\} \leqslant\{b\{t a t\} b\},
$$

and since $a, b, t \in\{u A u\}$ are compatible by centrality of $a$ and $b$, this gives (cf. [4; Lemma 2.11])

$$
b_{1} \leqslant U_{b} U_{t} a=U_{t} U_{b} a=0,
$$

a contradiction. This completes the proof that there is no nontrivial central idempotent in $\{u A u\}$, so $\{u A u\}$ is a $J B W$-factor.

Recall from [4] that a $J B W$-factor of type $I$ is said to be of type $I_{2}$ if the maximal cardinality of a set of orthogonal non-zero idempotents is 2 (ef. $[4 ; \S 6]$ ).

LеммА 3.9. If $u, v$ are distinct minimal idempotents in a $J B W$-factor $A$, then $A_{u, v}=$ $\{(u \vee v) A(u \vee v)\}$ is a type $I_{2} J B W$-factor.

Proof. By Lemma 3.8, $A_{u, v}$ is a $J B W$-factor. Since $A_{u, v}$ contains the minimal idempotents $u$ and $v$, it must be of type $I$. Note also that the maximal number of elements in a set of orthogonal idempotents is at least 2, since $u$ and $u \vee v-u$ are orthogonal and both non-zero. On the other hand, suppose that $w_{1}, w_{2}, w_{3}$ were three non-zero orthogonal idempotents in $A_{u, v}$. Then $w_{1}+w_{2}$ would be a non-minimal idempotent under $u \vee v$, contrary to Lemma 3.7. Thus, there can not be any set of more than 2 orthogonal nonzero idempotents in $A_{u, v}$; so $A_{u, v}$ is an $I_{2}$-factor.

Lемма 3.10. If $A$ is a JBW-factor of type $I_{2}$, then all states of $A$ are normal and the state space of $A$ is affinely isomorphic to a Hilbert ball.

Proof. Let $A$ be any $J B W$-factor of type $I_{2}$, and note that by Proposition 7.1 of [4] $A$ will be an (abstract) spin factor in the sense of Topping [19], i.e. $A$ can be equipped with an inner product $(a \mid b)$ which makes it a real Hilbert space in such a way that $e$ is a unit vector and for every pair $a, b$ of elements of $N=\{e\}^{\perp}$ one has

$$
a \circ b=(a \mid b) e .
$$

In particular $\|a\|^{2}=\left\|a^{2}\right\|=|(a \mid a)|$, so the Hilbert norm $\|\mid a\|=\sqrt{(a \mid a)}$ will coincide with the given $J B$-algebra norm $\|a\|$ for $a \in N$. Clearly also the two norms will coincide on $\mathbf{R} e$. By Lemmas 3 and 4 of [19] the following inequalities will hold for a general element $a \in A$ 


$$
\||a\|\leqslant\| a\|\leqslant \sqrt{2}|\|a \mid\| .
$$

Therefore $A$ is linearly homeomorphic to a Hilbert space, and so is reflexive. Hence the predual of $A$ will coincide with its dual, and all states must be normal.

We will prove that the closed unit ball of the subspace $N$ of $A$ provided with the Hilbert norm $\||a|\|$, is affinely isomorphic to the state space of $A$. To this end we first note that by Lemma 1 of [19] a general element $a+\alpha e \in A$, with $a \in N$ and $\alpha \in \mathbf{R}$, is positive iff

$$
\alpha \geqslant\||| a\| \mid
$$

For every given $b \in N$ we define a linear functional $\varrho_{b}$ on $A$ by

$$
\varrho_{b}(a+\alpha e)=(a \mid b)+\alpha
$$

where $a \in N$ and $\alpha \in \mathbf{R}$

Clearly $b \mapsto \varrho_{b}$ is an affine map of $N$ into $A^{*}$. If ||$|b||| \leqslant 1$, then for every $a+\alpha e \geqslant 0$ with $a \in N$ and $\alpha \in \mathbf{R}$ we get by (3.10) and (3.11):

$$
\varrho_{b}(a+\alpha e) \geqslant \alpha-|(a \mid b)| \geqslant \alpha-|||a||| \geqslant 0 .
$$

Hence $\varrho_{b} \geqslant 0$ when ||$|b| \| \leqslant 1$. By the definition (3.11), $\varrho_{b}(e)=1$ for all $b \in N$. Thus, $b \mapsto \varrho_{b}$ is an affine map of the closed unit ball of $N$ into the state space of $A$.

Now let $b_{1}, b_{2}$ be two distinct vectors in $N$. Letting $a=b_{1}-b_{2} \neq 0$, we have by (3.11)

$$
\varrho_{b_{1}}(a)-\varrho_{b_{2}}(a)=\left\{a\left|b_{1}-b_{2}\right\rangle=\left\||| b_{1}-b_{2} \mid\right\|^{2} \neq 0 .\right.
$$

Hence the map $b \mapsto \varrho_{b}$ is $1-1$.

Finally we consider an arbitrary state $\varrho$ of $A$. Since the two norms on $A$ coincide on $N$, the restriction of $\varrho$ to $N$ will have norm at most 1 with respect to the Hilbert norm. Hence there exists $b \in N$ with $\||b|\| \mid \leqslant 1$ such that $\varrho(a)=(a \mid b)$ for all $a \in N$. By linearity

$$
\varrho(a+\alpha e)=(a \mid b)+\alpha \varrho(e)=(a \mid b)+\alpha
$$

for arbitrary $a \in N$ and $\alpha \in \mathbf{R}$. Hence $\varrho=\varrho_{b}$. This proves that the map $b \mapsto \rightarrow \varrho_{b}$ maps the unit ball of $N$ onto the state space of $A$, and we are done.

THEOR Е 3.11 . The normal state space of a JBW-algebra has the Hilbert ball property.

Proof. Let $A$ be any $J B W$-algebra with normal state space $K$. By Proposition 3.3 every extreme point of $K$ is norm-exposed. We consider an arbitrary pair $\varrho, \sigma$ of extreme points of $K$ which can not be separated by a split face, and we will prove that face $(\{\varrho, \sigma\})$ is a norm-exposed face affinely isomorphic to a Hilbert ball. By previous remarks this will prove our theorem. 
Let $R$ be the $P$-projection, and $w$ the projective unit, corresponding to the projective face $F(\{\varrho, \sigma\})$ generated by $\varrho$ and $\sigma$. Since $\{\varrho\}$ and $\{\sigma\}$ are norm-exposed faces, they will also be projective faces. We denote the corresponding (minimal) projective units by $u$ and $v$, respectively. In the lattice $₹$ of projective faces of $K$, we have $F(\{\varrho, \sigma\})=$ $\{\varrho\} \vee\{\sigma\}$. Hence in the lattice $u$ of projective units, we have $w=u \vee v$. Therefore $R$ is the $P$-projection corresponding to $u \vee v$, and so $R=U_{u \vee v}$. Thus, for $a \in A$

$$
R a=\{(u \vee v) a(u \vee v)\}
$$

We write $A_{u, v}=\operatorname{im} R$, and we note that $A_{u, v}$ is a $J B W$-algebra by [4; Prop. 4.11]. By [3; Prop. 1.10], (im $\left.R^{*}, F(\{\varrho, \sigma\})\right)$ will be the predual of $\left(A_{u, v}, u \vee v\right)$. Thus $F(\{\varrho, \sigma\})$ will be the normal state space of $A_{u, v}$.

Now, let $c(\varrho)$ be the smallest central projective unit of $A$ such that $\langle c(\varrho), \varrho\rangle=1$, and recall that the corresponding projective face $G$ is the smallest split face containing $\varrho$. Since the two extreme points $\varrho$ and $\sigma$ can not be separated by a split face, then also $\sigma \in G$. It follows that $F(\{\varrho, \sigma\}) \subseteq G$, and by passage to projective units, $u \vee v \leqslant c(\varrho)$.

Let $Q$ be the (central) $P$-projection corresponding to $c(\varrho)$, let $A_{c(\varrho)}=\operatorname{im} Q$, and note that $u \in A_{c(Q)}$ and $v \in A_{c(Q)}$. (Recall that by [2; Cor. 2.12], im $Q$ is the order ideal of $A$ generated by $c(\varrho)$.) By Lemma 5.5. of [4], $A_{c(\varrho)}$ is a $J B W$-factor; and since it contains the minimal idempotents $u$, $v$, it must be of type $\mathrm{I}$.

Next we note that $A_{u, v}=R A=R A_{c(\varrho)}$ since $A_{u, v} \subseteq A_{c(\varrho)}$. Then it follows from Lemma 3.9 and formula (3.12) that $A_{u, v}$ is an $I_{2}$-factor. By Lemma 3.10 the normal state space $F(\{\varrho, \sigma\})$ of $A_{u, v}$ must be a Hilbert ball.

Finally we note that the face generated by any pair of distinct extreme points of a Hilbert ball, is the entire ball. Hence face $(\{\varrho, \sigma\})=F(\{\varrho, \sigma\})$. Thus, face $(\{\varrho, \sigma\})$ is a projective, hence norm-exposed, face of $K$ affinely isomorphic to a Hilbert ball.

CoRollany 3.12. The state space of a JB-algebra has the Hilbert ball property.

Proof. The state space of a $J B$-algebra is the normal state space of its enveloping $J B W$-algebra (cf. [17]), so Theorem 3.11 applies.

Now we turn to discuss a second geometric property which will be useful in the sequel. First we define a reflection of a convex set $K$ (in some linear space) to be an affine automorphism $\varphi$ of $K$ which is involutory (i.e. $\varphi^{2}=\mathrm{id}$ ). To justify the term "reflection", note that such a $\varphi$ has a non-empty set of fixed points $K_{0}=\left\{\frac{1}{2}(\varrho+\varphi(\varrho)) \mid \varrho \in K\right\}$, and that for each $\varrho \in K$ the image point $\varphi(\varrho)$ is obtained by reflecting the line segment $[\varrho, \varphi(\varrho)]$ about its mid-point $\frac{1}{2}(\varrho+\varphi(\varrho)) \in K_{0}$. 
Definition. A convex set $K$ is symmetric with respect to a convex subset $K_{0}$ if there exists a reflection of $K$ whose set of fixed points is precisely $K_{0}$.

LEMMA 3.13. Let $(A, e)$ be an order-unit space which is the dual of a base-norm space $(V, K)$. If $F$ is a projective face of $K$ and $P$ is the corresponding $P$-projection on $A$, then the following are equivalent:

(i) $K$ is symmetric with respect to co $\left(F \cup F^{*}\right)$

(ii) $2 P+2 P^{\prime}-I \geqslant 0$.

If these equivalent conditions hold, then $\varphi=\left.\left(2 P+2 P^{\prime}-I\right)^{*}\right|_{K}$ is the unique reflection of $K$ with co $\left(F \cup F^{\#}\right)$ as its set of fixed points.

Proof. Assume first that (i) holds, and let $\varphi$ be a reflection of $K$ with co $\left(F \cup F^{*}\right)$ as its set of fixed points. Define $\psi=\frac{1}{2}(\varphi+\mathrm{id})$, and note that $\psi(K) \subset K$ and $\psi^{2}=\psi$. Furthermore, for $\varrho \in K$ it is easily verified that $\psi(\varrho)=\varrho$ iff $\varphi(\varrho)=\varrho$, so $\psi(K)=\operatorname{co}\left(F \cup F^{*}\right)$. Thus $\psi$ is an affine retraction of $K$ onto co $\left(F \cup F^{*}\right)$. By [2; Th. 3.8] there exists exactly one affine retraction of $K$ onto co $\left(F \cup F^{*}\right)$, namely $\left.\left(P+P^{\prime}\right)^{*}\right|_{K}$. Thus we have $\psi=\left.\left(P+P^{\prime}\right)^{*}\right|_{K}$, which implies $\varphi=\left.\left(2 P+2 P^{\prime}-I\right)^{*}\right|_{K}$. In particular $\left(2 P+2 P^{\prime}-I\right)^{*}$ leaves the base $K$ of the cone $V+$ invariant. Hence $2 P+2 P^{\prime}-I$ leaves the cone $A^{+}$invariant, so (ii) is proven. In addition we have proven the uniqueness statement of the lemma.

Assume next that (ii) holds. Then since $2 P+2 P^{\prime}-I \geqslant 0$ and $\left(2 P+2 P^{\prime}-I\right) e=e$, the dual map $\left(2 P+2 P^{\prime}-I\right)^{*}$ will leave invariant not only the cone $V+$ but also its base $K$. Since $\left(2 P+2 P^{\prime}-I\right)^{2}=I$, the map $\left.\left(2 p+2 p^{\prime}-I\right)^{*}\right|_{K}$ is a reflection of $K$. Furthermore $\left(2 P+2 P^{\prime}-I\right)^{*} \varrho=\varrho$ iff $\left(P+P^{\prime}\right)^{*} \varrho=\varrho$, which by [2; Th. 3.8]) is equivalent to $\varrho \in \operatorname{co}\left(F^{\prime} \cup F^{*}\right)$. This shows that $K$ is symmetric with respect to co $\left(F \cap F^{*}\right)$, which completes the proof.

Proposition 3.14. The normal state space of a JBW-algebra is symmetric with respect to co $\left(F \cup F^{*}\right)$ for every projective face $F$, or what is equivalent:

$$
2 P+2 P^{\prime}-I \geqslant 0 \text { for all } P \in D \text {. }
$$

Proof. Let $A$ be a $J B W$-algebra with normal state space $K$.

We will first verify the following identity valid for any idempotent $q \in A$ with $q^{\prime}=e-q$ :

$$
\left\{\left(q-q^{\prime}\right) a\left(q-q^{\prime}\right)\right\}=2\{q a q\}+2\left\{q^{\prime} a q^{\prime}\right\}-a .
$$

By definition, $\{b a b\}=2 b \circ(b \circ a)-b^{2} \circ a$ for any $b \in A$. Applying this to $b=q-q^{\prime}=$ $2 q-e$, we find

$$
\left\{\left(q-q^{\prime}\right) a\left(q-q^{\prime}\right)\right\}=4\{q a q\}-4 q \circ a+a
$$


Interchanging the roles of $q$ and $q^{\prime}$, we get

$$
\left\{\left(q-q^{\prime}\right) a\left(q-q^{\prime}\right)\right\}=4\left\{q^{\prime} a q^{\prime}\right\}-4 q^{\prime} \circ a+a .
$$

Addition of (3.15) and (3.16) gives (3.14).

Now suppose $P$ is any $P$-projection on $A$. Then $P=U_{q}, P^{\prime}=U_{q^{\prime}}$ for some idempotent $q \in A$. Thus (3.14) gives the following equality valid for each $a \in A$ :

$$
\left(2 P+2 P^{\prime}-I\right) a=\left(2 U_{q}+2 U_{q^{*}}-I\right) a=\left\{\left(q-q^{\prime}\right) a\left(q-q^{\prime}\right)\right\} .
$$

By Proposition 2.7 of [4] the maps $U_{b}: a \mapsto\{b a b\}$ are positive for all $b$, and so we conclude $2 P+2 P^{\prime}-I \geqslant 0$, which proves $(3.13)$.

Passing from a $J B$-algebra to its enveloping $J B W$-algebra, we also obtain:

COROLLARY 3.15. The state space of a JB-algebra is symmetric with respect to co $\left(F \cup F^{*}\right)$ for every projective face $F^{\prime}$.

Remark. In the proof above $s=q-q^{\prime}$ satisfies $s^{2}=e$. Thus each map $2 P+2 P^{\prime}-I$ in the $J B$-algebra context is of the form $U_{s}$ for some element $s$ such that $s^{2}=e$. In the operator algebra context the map $U_{s}$ is conjugation by the self-adjoint unitary $s$. Thus symmetry of the state space for a $C^{*}$-algebra can be viewed as a consequence of the fact that conjugation by a self-adjoint unitary is an involutary *-automorphism, and thus induces a reflection of the state space.

\section{\$4. The pure state properties}

Throughout this section we will assume that $(A, e)$ is an order-unit space which is the dual of a base-norm space $(V, K)$ and that (2.5) holds. The extreme rays of $V^{+}$are the sets $\mathbf{R}^{+} \varrho$ with $\varrho$ an extreme point of $K$. Thus, to say that a map $P^{*}$ (with $P \in \mathcal{D}$ ) preserves extreme rays, means that $P^{*} \varrho$ is a multiple of an extreme point for every extreme point $\varrho$ of $K$. (Here we allow the possibility that $P^{*} \varrho=0$.)

Proposition 4.1. If $K$ has the Hilbert ball property and is symmetric with respect to co $\left(F \cup F^{*}\right)$ for every projective face $F$, then $P^{*}$ preserves extreme rays for every $P \in \emptyset$.

Proof. Let $\varrho \in K$ be an arbitrary extreme point. We can, and shall, assume $\left(P+P^{\prime}\right)^{*} \varrho \neq \varrho$; for otherwise $0 \leqslant P^{*} \leqslant \varrho$, and then $P^{*} \varrho$ would be a multiple of the extreme point $\varrho$.

By the symmetry property, the map $\left(2 P+2 P^{\prime}-I\right)^{*}$ acts as an automorphism of $K$. Hence $\omega=\left(2 P+2 P^{\prime}-I\right)^{*} \varrho$ is an extreme point of $K$. By the ball property, face $(\{\varrho, \omega\})$ is affinely isomorphic to a Hilbert ball. 
Now let $\lambda=\left\|P^{*} \varrho\right\| \neq 0$, and observe that $1-\lambda=\left\|P^{* *} \varrho\right\| \neq 0$. Define also $\sigma=\left\|P^{*} \varrho\right\|^{-1} P^{*} \varrho$ and $\tau=\left\|P^{\prime *} \varrho\right\| P^{* *} \varrho$. Then

$$
\frac{1}{2}(\varrho+\omega)=\left(P+P^{\prime}\right)^{*} \varrho=\lambda \sigma+(1-\lambda) \tau .
$$

Now it follows from the definition of a face that $\sigma, \tau \in$ face $(\{\varrho, \omega\})$.

The points $\sigma$ and $\tau$ are contained in disjoint faces $K \cap \operatorname{im} P^{*}$ and $K \cap \operatorname{im} P^{\prime *}$; therefore the line segment $[\sigma, \tau]$ can not be extended beyond $\sigma$ or $\tau$ within $K$. Thus, $\sigma$ and $\tau$ must be boundary points, and then also extreme points of the ball face $(\{\varrho, \omega\})$. It follows that $\sigma=\left\|P^{*} \varrho\right\|^{-1} P^{*} \varrho$ is an extreme point of the given convex set $K$, and the proof is complete.

In our next proposition we will introduce two properties which are consequences of the preservation of extreme rays by $P^{*}$ for $P \in D$. In fact, under the assumption that every extreme point of $K$ is exposed, it is not hard to verify that each one of them is equivalent with the preservation of extreme rays. However, this will not be needed in the sequel, so we omit the proof.

Proposition 4.2. If $K$ is such that $P^{*}$ preserves extreme rays for every $P \in \mathcal{D}$, then:

(i) For each atom $u \in \mathcal{U}$ and each $v \in \mathcal{U},\left(u \vee v^{\prime}\right) \wedge v$ is either an atom or zero.

(ii) Each $P \in \mathcal{D}$ maps atoms to multiples of atoms.

Proof. 1. Let $Q$ be the $P$-projection and $G$ the projective face corresponding to $v \in \mathcal{U}$, and recall that $\{\hat{u}\}$ is the projective face corresponding to the atom $u \in \mathcal{U}$. By [3; Prop. 1.9]

$$
F\left(Q^{*} \hat{u}\right)=\left(\{\hat{u}\} \vee G^{*}\right) \cap G
$$

By hypothesis $Q^{*} \hat{u}$ is either zero or a multiple of an extreme point. In the latter case $\left\{\left\|Q^{*} \hat{u}\right\|^{-1} Q^{*} \hat{u}\right\}$ is a norm-exposed, hence projective, face of $K$. Therefore $\left\|Q^{*} \hat{u}\right\|^{-1} Q^{*} \hat{u}=\hat{\omega}$ for some atom $w \in \mathcal{U}$. Now (4.1) can be rewritten in the form $\{\hat{\omega}\}=\left(\{\hat{u}\} \vee G^{*}\right) \cap G$. By the isomorphism of the lattice $\mathcal{U}$ of projective units and the lattice $₹$ of projective faces, this gives the equality $w=\left(u \vee v^{\prime}\right) \wedge v$, which completes the proof of (i).

2. Let $P \in \mathcal{D}$ and let $u \in \mathcal{U}$ be an atom. By [3; Lemma 1.8]

$$
r(P u)=\left(u \vee P^{\prime} e\right) \wedge P e,
$$

and by statement (i) the right term is either zero or an atom. Therefore $P u$ must be a multiple of an atom.

Remarks. As was first observed by Pool in a related context [14], the statement (i) of Proposition 4.2 is closely related to the notion of semimodularity of the lattice $\mathcal{U}$. It is well known that for every orthomodular lattice semimodularity implies (i), and it can 
be proved that the converse implication holds if there are "sufficiently many" atoms, i.e. if every element of the lattice is l.u.b. of atoms. (See [15] and [11; Th. 30.2, Cor. 7.7].)

Proposition 4.3. If $K$ has the Hilbert ball property, then for every pair of atoms $u, v \in \mathcal{U}:$

$$
\langle u, \hat{v}\rangle=\langle v, \hat{u}\rangle
$$

Proof. By hypothesis $F=$ face $(\{\hat{u}, \hat{v}\})$ is a norm-exposed face affinely isomorphic to a Hilbert ball. Being norm-exposed, the face $F$ is also projective, therefore of the form $F=K \cap \operatorname{im} Q^{*}$ for $Q \in \mathcal{D}$. Since $\hat{u}, \hat{v} \in F$, we can pass to the corresponding projective units and obtain $u, v \leqslant Q e$. Now by [3; Prop. 1.10], $u$ and $v$ will de:ermine projective units in the relativized spectral duality of $(\operatorname{im} Q, Q e)$ and $\left(\operatorname{im} Q^{*}, F^{\prime}\right)$. Thus the restriction of $u$ and $v$ to $F$ will be affine functions with values in $[0,1]$; the former with the values 1 at $\hat{u}$ and 0 at its antipodal point, and the latter with the values 1 at $\hat{v}$ and zero at its antopodal point.

Denoting by $\pi$ the center of the ball $F$ and by $\alpha$ the angle between $\hat{u}-\pi$ and $\hat{v}-\pi$ and using some elementary plane geometry, we obtain (see fig. 1):

$$
\langle u, \hat{v}\rangle=\langle v, \hat{u}\rangle=\frac{1}{2}(1+\cos \alpha) \text {. }
$$

Note that this proof is valid also when $F$ is one-dimensional: Then $\alpha=180^{\circ}$ and $\langle u, \hat{v}\rangle=$ $\langle v, \hat{u}\rangle=0$.

The properties established in Propositions 4.1 and 4.3, will always be used in connection with the property that all extreme points of $K$ are norm-exposed. It will be convenient to be able to refer to them by a single name; since they are all related to the extreme points of $K$, we give the following:

Definition. $K$ has the pure state properties if:

Every extreme point of $K$ is norm-exposed.

$$
P^{*} \text { preserves extreme rays for every } P \in \mathfrak{D} \text {. }
$$

$$
\langle u, \hat{v}\rangle=\langle v, \hat{u}\rangle \text { for every pair of atoms } u, v \in \mathcal{U} \text {. }
$$

Remarks on the physical interpretation of the pure state properties. As in $[3 ; \S 3]$ we may view each $\varrho \in V^{+}$as representing a beam of particles with intensity $\|\varrho\|=\langle e, \varrho\rangle$, and each $P \in D$ as representing a filter transforming any given beam $\varrho \in V^{+}$to a new beam $P^{*} Q$ of intensity at most equal to that of the given beam. (By a fundamental property of $P$-projections, the intensity remains undiminished only when the filter is neutral to the beam, cf. $[2 ; \S 2]$. 


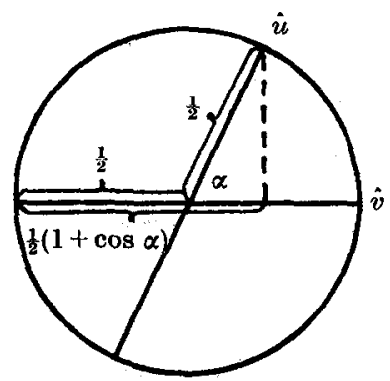

Fig. 1

Property (4.3) states that for an arbitrary beam of particles in a pure state, i.e. for an arbitrary extreme point $\varrho$ of $K$, there exists a filter $P \in \mathcal{D}$ which transforms every incoming beam to (a multiple of) the given beam, i.e. $P^{*}\left(V^{+}\right)=\mathbf{R}+\varrho$. (This may be expressed by saying that the filter $P$ "prepares" $\varrho$.)

Property (4.4) states that each filter $P \in \mathcal{D}$ transforms pure states to (scalar multiples of) pure states. (The scalar factor represents the decrease in intensity, and is strictly less than 1 unless $P^{*} e=\varrho$, as noted above.)

Property (4.5) is a statement of a symmetric relationship between the "transition probabilities" connecting pure states. For a more detailed discussion of these and related properties see [14], [7], [12]:

\section{§ 5. The splitting into atomic and non-atomic parts}

Until further notice we will make the same hypotheses as in $\$ 4$, i.e. we assume that $(A, e)$ and $(V, K)$ are given such that $A=V^{*}$ and $(2.5)$ holds. We will first present some relevant lattice theoretic definitions and results, which we state for the (complete) orthomodular lattice $\mathcal{U}$ of projective units in $A$.

Let $u, v, w \in \mathcal{U}$, we say $v$ covers $u$ if $u<v$ and $u \leqslant w \leqslant v$ implies $w=u$ or $w=v$. Clearly this is equivalent with $v-u$ being an atom. We say that $u$ has the covering property if

$$
u, v \in U \text { and } v \text { an atom implies } u \vee v \text { covers or equals } u \text {. }
$$

Note that (5.1) holds in the particular case where $\mathcal{U}$ is the lattice of idempotents in a $J B W$-algebra (Lemma 3.6).

An element $u \in \mathcal{U}$ is said to be finite if it is the 1.u.b. of a finite number of atoms; the minimum number of atoms whose l.u.b. is $u$, is called the (lattice theoretic) dimension of $u$. We say $\mathcal{U}$ has the finite covering property if (5.1) holds for all finite $u \in U$. Also we say $\mathcal{U}$ is atomic if every element of $\mathcal{U}$ is the l.u.b. of an orthogonal set of atoms. In the sequel we will use the symbol $A_{f}$ to denote the linear span in $A$ of all atoms of $U$.

12- 772908 Acta mathematica 140. Imprimé Je 9 Juin 1978 
LEмма 5.1. Assume that $\mathcal{U}$ is atomic and has the finite covering property. If $u \in \mathcal{U}$ is finite, then every $v \leqslant u$ is finite with $\operatorname{dim} v \leqslant \operatorname{dim} u$. Furthermore, every set of mutually orthogonal non-zero $v \in \mathcal{U}$ such that $v \leqslant u$, has cardinality at most equal to $\operatorname{dim} u$.

Proof. The result will follow from [10; Th. 4.1]; we only have to show that if $u, v \in \mathcal{U}$ and $u$ and $v$ both cover $u \wedge v$, then $u \vee v$ covers $u$ and $v$. Since $x \vdash x-(u \wedge v)$ is an order isomorphism of the lattice interval $[u \wedge v, u \vee v]$ onto $[0, u \vee v-u \wedge v]$, we can assume $u \wedge v=0$ without loss of generality. Then $u$ and $v$ are atoms, so $u \vee v$ covers $u$ and $v$ by the finite covering property.

From now onward we specialize to the case of a $J B W$-algebra $A$ in duality with its predual $V$, with $K$ being the normal state space of $A$. We will say $A$ is atomic if the lattice $U$ of projective units (=idempotents) is atomic.

LEMMA 5.2. If $A$ is a $J B W$-algebra, then $A$ is the direct sum of an atomic $J B W$-algebra and a $J B W$-algebra containing no atoms.

Proof. Using the techniques of [4] we can apply the proof of [18; Prop. 1.3]. (In $\$ 6$ we shall give a direct proof of this result in a more general context.)

Proposition 5.3. If $A$ is a $J B W$-algebra, then every $a \in A_{f}$ can be written as a finite linear combination $a=\sum_{i=1}^{n} \lambda_{i} u_{i}$ of orthogonal atoms $u_{1}, \ldots, u_{n}$.

Proof. By Lemma 5.2 we may assume that $A$ is atomic. Let $a=\sum_{i=1}^{n} \lambda_{i} v_{i}$ where $v_{1}, \ldots, v_{n}$ are atoms. Define $v=v_{1} \vee \ldots \vee v_{n}$ and $A_{v}=\{v A v\}$. Then $A_{v}$ is an atomic $J B W$-algebra, and by Lemma 5.1 there is a finite upper bound on the cardinality of orthogonal sets of idempotents in $A_{v}$. The desired representation of a now follows from the spectral theorem in $A_{v}$.

LEMMA 5.4. If $A$ is an atomic $J B W$-algebra, then its normal state space $K$ is the norm closed convex hull of its extreme points.

Proof. It clearly suffices to prove that the cone generated by the set of extreme points $\partial_{e} K$ is norm dense in $V^{+}$. To this end we suppose $a \in A$ and $\langle a, \varrho\rangle \geqslant 0$ for all $\varrho \in \partial_{e} K$, and we will show that $a \in A^{+}$. By a standard Hahn-Banach argument, this will complete the proof.

Choose $P \in \mathcal{D}$ such that $P a=-a^{-}$(cf. $\S 2$ ). For each $\varrho \in \partial_{e} K, P^{*} \varrho$ will be a non-negative multiple of an extreme point (Proposition 4.1), so

$$
0 \leqslant\left\langle a^{-}, \varrho\right\rangle=-\langle P a, \varrho\rangle=-\left\langle a, P^{*} \varrho\right\rangle \leqslant 0 .
$$

Thus $a^{-}$annihilates $\partial_{e} K$. By hypothesis, the lattice $\mathcal{F}(\cong \mathcal{U})$ of projective faces of $K$ is atomic; therefore the norm-exposed (=projective) face generated by $\partial_{e} K$ is $K$ itself. Hence $a^{-}=0$, and so $a=a^{+} \geqslant 0$. 
LEMMA 5.5. If $A$ is an atomic $J B W$-algebra, then there exists an order isomorphism $\psi$ from the predual $V$ of $A$ of $A$ into $A$ itself such that $\|\psi\| \leqslant 1$ and $\psi(\hat{u})=u$ for all atoms $u$ in $A$.

Proof. We first observe that if $u_{1}, \ldots, u_{n}$ are atoms such that $\sum_{i=1}^{n} \lambda_{i} \hat{u}_{i} \geqslant 0$ for given constants $\lambda_{1}, \ldots, \lambda_{n}$, then by the pure state property (4.5)

$$
\left\langle\sum_{i=1}^{n} \lambda_{i} u_{i}, \hat{v}\right\rangle=\left\langle v, \sum_{i=1}^{n} \lambda_{i} \hat{u}_{i}\right\rangle \geqslant 0
$$

for each atom $v$; hence $\sum_{i=1}^{n} \lambda_{i} u_{i} \geqslant 0$ by Lemma 5.4. Thus, there exists a well defined positive linear map $\psi_{0}: \operatorname{lin}\left(\partial_{e} K\right) \rightarrow A$ given by

$$
\psi_{0}\left(\sum_{i=1}^{n} \lambda_{i} \hat{u}_{i}\right)=\sum_{i=1}^{n} \lambda_{i} u_{i}
$$

Clearly $\psi_{0}$ maps $\operatorname{lin}\left(\partial_{e} K\right)$ onto $A_{f}$. To prove injectivity, let $\psi_{0}(\varrho)=0$ where $\varrho=\sum_{i=1}^{n} \lambda_{i} \hat{u}_{i}, u_{1}, \ldots, u_{n}$ being atoms. By the pure state property (4.5) once more

$$
0=\left\langle\psi_{0}(\varrho), \hat{v}\right\rangle=\left\langle\sum_{i=1}^{n} \lambda_{i} u_{i}, \hat{v}\right\rangle=\left\langle v, \sum_{i=1}^{n} \lambda_{i} \hat{u}_{i}\right\rangle=\langle v, \varrho\rangle
$$

for all atoms $v$. Hence $\varrho=0$ by spectral theory, atomicity of $A$ and [2; Lemma 12.1]. Thus, $\psi_{0}$ is injective.

Next we show $\left\|\psi_{0}\right\| \leqslant 1$. By Proposition 5.3 each $\varrho \in \operatorname{lin}\left(\partial_{e} K\right)$ admits a representation $\varrho=\sum_{i=1}^{n} \lambda_{i} \hat{u}_{i}$ where $u_{1}, \ldots, u_{n}$ are orthogonal atoms. It is not difficult to verify that $\left.\|e\|=\sum_{i=1}^{n} \mid \lambda_{i}\right\}$, and so

$$
\left\|\psi_{0}(\varrho)\right\|=\left\|\sum_{i=1}^{n} \lambda_{i} u_{i}\right\|=\max \left|\lambda_{i}\right| \leqslant \sum_{i=1}^{n}\left|\lambda_{i}\right|=\|\varrho\|
$$

Thus, $\left\|\psi_{0}\right\| \leqslant 1$.

By density of lin $\left(\partial_{e} K\right)$ in $V$ (Lemma 5.4) and by completeness of $A$, we can extend $\psi_{0}$ to a positive linear map $\psi: V \rightarrow A$ such that $\|\psi\| \leqslant 1$. Observe also that by the pure state property $(4.5)$, we have

$$
\langle\psi(\varrho), \sigma\rangle=\langle\psi(\sigma), \varrho\rangle
$$

for all $\varrho, \sigma \in \partial_{e} K$; by linearity and continuity (5.2) will hold for all $\varrho, \sigma \in V$.

Finally, to show $\psi$ bipositive we suppose $\varrho \in V$ and $\psi(\varrho) \geqslant 0$. By $(5.2)\langle\psi(\sigma), \varrho\rangle \geqslant 0$ for all $\sigma \in V^{+}$, and in particular for $\sigma \in \partial_{e} K$. By spectral theory and atomicity of $A$, we get $\varrho \geqslant 0$. This completes the proof.

Proposition 5.6. If $A$ is an atomic $J B W$-algebra, then every $\varrho \in K$ can be expressed as $\varrho=\sum_{i=1}^{\infty} \lambda_{i} \varrho_{i}$ where $\sum_{i=1}^{\infty} \lambda_{i}=1, \lambda_{i} \geqslant 0$ and $\varrho_{i} \in \partial_{e} K$ for all $i$, and $\varrho_{i} \perp \varrho_{j}$ for $i \neq j$. 
Proof. Let $\varrho \in K$. For given $\alpha>0$ we have by the spectral theorem in $A$

$$
\psi(\varrho)=\int_{0}^{\infty} \lambda d e_{\lambda} \geqslant \alpha\left(e-e_{\alpha}\right)
$$

Suppose $u_{1}, \ldots, u_{n}$ are orthogonal atoms below $e-e_{\alpha}$, then

$$
\psi(\varrho) \geqslant \alpha\left(e-e_{\alpha}\right) \geqslant \alpha\left(\sum_{i=1}^{n} u_{i}\right)=\alpha \psi\left(\sum_{i=1}^{n} \hat{u}_{i}\right)
$$

Since $\psi$ is an order isomorphism, then also $\varrho \geqslant \alpha \sum_{i=1}^{n} \hat{u}_{i}$, and so

$$
\mathrm{I}=\|\varrho\|^{2} \geqslant\langle\psi(\varrho), \varrho\rangle \geqslant \alpha^{2}\left\langle\sum_{i=1}^{n} u_{i}, \sum_{i=1}^{n} \hat{u}_{i}\right\rangle=n \alpha^{2}
$$

Thus $n \leqslant \alpha^{-2}$, ans so there are only a finite number of orthogonal atoms below $e-e_{\alpha}$.

It follows that

$$
\psi(\varrho)=\int_{0}^{\infty} \lambda d e_{\lambda}=\sum_{i=1}^{\infty} \lambda_{i} v_{i}
$$

where $v_{1}, v_{2}, \ldots$ are orthogonal atoms and $\lambda_{i} \searrow 0$. Observe that $\sum_{i=1}^{\infty} \lambda_{i} \leqslant 1$ since $\psi(\varrho) \geqslant$ $\sum_{i=1}^{n} \lambda_{i} v_{i}$ gives

$$
1=\langle e, \varrho\rangle \geqslant\left\langle e, \sum_{i=1}^{n} \lambda_{i} \hat{v}_{i}\right\rangle=\sum_{i=1}^{n} \lambda_{i}
$$

Thus $\sum_{i=1}^{\infty} \lambda_{i} \hat{v}_{i}$ is a norm convergent series such that

and so $\varrho=\sum_{i=1}^{\infty} \lambda_{i} \hat{v}_{i}$. Also

$$
\psi(\varrho)=\psi\left(\sum_{i=1}^{\infty} \lambda_{i} \hat{v}_{i}\right),
$$

$$
\mathrm{I}=\langle e, \varrho\rangle=\left\langle e, \sum_{i=1}^{\infty} \lambda_{i} \hat{v}_{i}\right\rangle=\sum_{i=1}^{\infty} \lambda_{i}
$$

which completes the proof.

For the statement of our next theorem, we recall that the $\sigma$-convex hull of a subset $E$ of $K$ consists of all (norm) convergent sums $\sum_{i=1}^{\infty} \lambda_{i} \varrho_{i}$ where $\sum_{i=1}^{\infty} \lambda_{i}=1$ and $\lambda_{i} \geqslant 0, \varrho_{i} \in K$ for all $i$.

THEOREM 5.7. Let $A$ be a $J B W$-algebra with normal state space $K$. Then the $\sigma$-convex hull of the extreme points of $K$ is a split face of $K$.

Proof. By Lemma 5.2 there is a central idempotent $c$ in $A$ such that $c \circ A$ is atomic and $(e-c) \circ A$ contains no atoms. Then $K \cap \operatorname{im} U_{c}^{*}$ and $K \cap \operatorname{im} U_{e-c}^{*}$ are complementary split faces of $K$, with the former being the normal state space of $c \circ A$ and the latter being the normal state space of $(e-c) \circ A$. By Proposition 5.6 the former is the $\sigma$-convex hull 
of its extreme points, and by the 1-1 correspondence of extreme points and atoms (cf. $\S 2$ and (4.3)) the latter contains no extreme points. This completes the proof.

COROLLARY 5.8. Let $K$ be the state space of a JB-algebra. Then the $\sigma$-convex hull of the extreme points of $K$ is a split face of $K$.

\section{§6. The type I factor case}

In this section we keep the assumptions of $\S 4$, thus we are given $(A, e)$ and $(V, K)$ such that $A=V^{*}$ and (2.5) holds. In addition we assume that $V$ is complete (in the base norm). Our purpose here is to find necessary and sufficient conditions for $K$ to be the normal state space of a $J B W$-factor of type $I$. An obvious necessary condition is that $A$ shall be a type I factor as defined in $\S 2$; we will show that one can obtain a set of necessary and sufficient conditions by adding either the Hilbert ball property and the splitting into atomic and non-atomic parts, or the pure state properties of $\S 4$. It will be convenient to start out from hypotheses which are implied by either of these sets of conditions, and to work in a context slightly more general than the type I factor case.

Thus, until further notice we will assume that $K$ satisfies the following requirements:

$$
\text { The lattice } \quad \mathcal{F}(\cong \mathcal{U}) \text { is atomic (cf. } \S 5 \text { ). }
$$

$\mathcal{I}$ has the finite covering property (cf. $\S 5$ ).

Every extreme point of $K$ is norm-exposed.

$$
\langle u, \hat{v}\rangle=\langle v, \hat{u}\rangle \quad \text { for each pair of atoms } u, v \in A \text {. }
$$

Observe that for each $P_{0} \in \mathcal{D}$ the above conditions (6.1)-(6.4) will also be satisfied in the relativized setting of $A_{0}=\operatorname{im} P_{0}$ and $V_{0}=\operatorname{im} P_{0}^{*}$, with $e_{0}=P_{0} e$ replacing $e$ and $K_{0}=K \cap V_{0}$ replacing $K$ (cf. [3; Prop. 1.10]).

Observe also that (6.3) and (6.4) are nothing but the pure state properties (4.3) and (4.5). We will now prove that a weak form of the remaining pure state property (4.4) will follow from our present assumptions. In this connection we define an element $P$ of the lattice $\mathcal{P}$ to be cofinite if $P^{\prime}$ is finite (similarly for $\mathcal{F}$ and $\mathcal{U}$ ). Recall also that if $P \in \mathcal{D}$ is finite, then every $Q \precsim P$ is finite by virtue of Lemma 5.1.

Lemma 6.1. $P^{*}$ preserves extreme rays if $P \in \mathcal{I}$ is finite or cofinite.

Proof. 1. Let $P \in \mathcal{F}$ be cofinite, say $P=R^{\prime}$ where $R$ is finite. Let $F=K \cap$ im $P^{*}$ be the projective face corresponding to $P$. Then $G=F^{*}$ corresponds to $R$. Following the terminology of [3], we denote the projective face of $K$ generated by a point $\sigma \in V^{+}$by $F(\sigma)$. 
For each $\varrho \in \partial_{e} K$, then $F(\varrho)=\{\varrho\}$ is a minimal element of $\mathcal{F}$. Since $G$ is finite in $\mathcal{F}$, the finite covering property implies that $F(\varrho) \vee G$ covers or equals $G$. This means that $(F(\varrho) \vee G) \wedge G^{*}$ is a minimal element of $\mathcal{F}$ or the empty set. By [3; Prop. 1.9] we have

$$
F\left(P^{*} \varrho\right)=\left(F^{\prime}(\varrho) \vee F^{*}\right) \cap F=(F(\varrho) \vee G) \wedge G^{*} ;
$$

hence $P^{*} \varrho$ is either a non-zero element located on an extreme ray in $V^{+}$, or $P^{*} \varrho=0$ (cf. [3; Prop. 1.13]). With this we have shown that for the cofinite $P$-projection $P$, then $P^{*}$ preserves extreme rays.

2. Keeping the notation from the first part of the proof, we will prove that for the finite $P$-projection $R$, then $R^{*}$ preserves extreme rays. For given $\varrho \in \partial_{e} K$, let $P_{e}$ be the $P$-projection corresponding to $F(\varrho)=\{\varrho\}$. Then $P_{0}=R \vee P_{Q}$ is finite. In the relativized setting of $A_{0}=\operatorname{im} P_{0}$ and $V_{0}=\operatorname{im} P_{0}^{*}$ the $P$-projections are (up to canonical identification, cf. [3; Prop. 1.10]) exactly those $Q \in \mathcal{F}$ which satisfy $Q \precsim P_{0}$; hence they are all finite. From the first part of the proof it follows that $R^{*} \varrho$ is a multiple of an extreme point in $K_{0}=K \cap \mathrm{im} P_{0}^{*}$. Since $K_{0}$ is a face of $K$, this point must be extreme in $K$ as well. Hence $R^{*} \varrho$ is a multiple of an extreme point in $K$, and we are done.

Lемма 6.2. If $\varrho \in V$ and $\langle v, \varrho\rangle \geqslant 0$ for all atoms $v \in A$, then $\varrho \geqslant 0$.

Proof. By [3; Prop. 1.7], $\mathcal{U}$ is a $w^{*}$-dense subset of $\partial_{e}[0, e]$, so by the Krein-Milman theorem it suffices to prove $\langle u, \varrho\rangle \geqslant 0$ for an arbitrary $u \in U$. By atomicity, $u$ will be the 1.u.b. of a set of orthogonal atoms, and by [2; Prop. 4.4, Lemma 12.1] the net of all finite sums of these atoms will converge to $u$ in the $w^{*}$-topology. Hence $\langle u, \varrho\rangle \geqslant 0$, as desired.

COROLLARY 6.3. $A_{f}$ and $V$ are in separating order and norm duality $(c f .[2 ; \S 1]$ for the definition).

Proof. $A_{f}$ and $V$ are in separating order duality by Lemma 6.2. To establish separating norm duality, we consider an element $\varrho \in V$ such that $|\langle a, \varrho\rangle| \leqslant 1$ for all $a \in A_{f}$ with $\|a\| \leqslant 1$, and we will show that $\|\varrho\| \leqslant 1$. Since $U$ is $w^{*}$-dense in $\partial_{e} K$, an elementary argument shows that $S=\{2 u-e \mid u \in U\}$ is $w^{*}$ dense in $\partial_{e}[-e, e]$ (cf. e.g. the proof of [3; Prop. 1.7]). Thus, by the Krein-Milman theorem it suffices to show that $|\langle 2 u-e, \varrho\rangle| \leqslant 1$ for an arbitrary $u \in \mathcal{U}$. As in the proof of Lemma 6.2, we construct nets $\left\{u_{\alpha}\right\}$ and $\left\{v_{\alpha}\right\}$ in $A_{f}$ such that $0 \leqslant u_{\alpha} \leqslant u, 0 \leqslant v_{\alpha} \leqslant e-u$ and $u_{\alpha} \rightarrow u, v_{\alpha} \rightarrow e-u$ in the $w^{*}$-topology. Then $u_{\alpha}-v_{\alpha} \in A_{f} \cap[-e, e]$ and $u_{\alpha}-v_{\alpha} \rightarrow 2 u-e$ in the $w^{*}$-topology. Hence $|\langle 2 u-e, \varrho\rangle| \leqslant 1$, as desired.

LEMMA 6.4. There exists a unique positive linear map $\varphi: A_{f} \rightarrow V$ such that $\varphi(u)=\hat{u}$ for all atoms $u$; the equation $(a \mid b)=\langle a, \varphi(b)\rangle$ will define a symmetric bilinear form on $A_{f}$ such that 


$$
\langle u \mid v\rangle=\langle u, \hat{v}\rangle
$$

for all pairs of atoms $u, v$; furthermore, each finite or cofinte $P \in \mathcal{D}$ maps $A_{f}$ into itself and satisfies the symmetry condition.

$$
(P a \mid b)=(a \mid P b)
$$

for all $a, b \in A_{f}$.

Proof. Observe first that if $u_{1}, \ldots, u_{n}$ are atoms such that $\sum_{i=1}^{n} \lambda_{i} u_{i} \geqslant 0$ for given constants $\lambda_{1}, \ldots, \lambda_{n}$, then by (6.4)

$$
\left\langle v, \sum_{i=1}^{n} \lambda_{i} \hat{u}_{i}\right\rangle=\left\langle\sum_{i=1}^{n} \lambda_{i} u_{i}, \hat{v}\right\rangle \geqslant 0
$$

for each atom $v$; hence $\sum_{i=1}^{n} \lambda_{i} \hat{u}_{i} \geqslant 0$ by Lemma 6.2 . Thus there exists a well defined positive linear map $\varphi: A_{f} \rightarrow V$ given by

$$
\varphi\left(\sum_{i=1}^{n} \lambda_{i} u_{i}\right)=\sum_{i=1}^{n} \lambda_{i} \hat{u}_{i}
$$

The bilinearity of the form $(a \mid b)=\langle a, \varphi(b)\rangle$ is trivial; so is (6.5), and the symmetry follows from (6.7) and (6.4).

Assume now that $P$ is finite or cofinite. By Lemma 6.1 and Proposition 4.2, $P$ maps atoms into multiples of atoms; hence $P\left(A_{f}\right) \subseteq A_{f}$. In order to prove that $P$ is symmetric with respect to the bilinear form on $A_{f}$, we start by establishing

$$
((I-P) u \mid P v)=0
$$

for an arbitrary pair of atoms $u, v$.

As remarked above, $P v$ is a multiple of an atom, say $P v=\lambda w$ where $\lambda \in \mathbf{R}^{+}$and $w=Q e$ for a $P$-projection $Q$ with one-dimensional range (cf. [3; Prop. 1.13]). Now, $\mathrm{im}^{+} Q$ is the ray determined by $w$, and since $w \in \mathrm{im}^{+} P$ we must have $\mathrm{im}^{+} Q \subseteq \mathrm{im}^{+} P$, which shows that the projective face corresponding to $Q$ is contained in the projective face corresponding to $P$ [2; Lemma 2.16]. By definition, $\hat{w}$ is the unique element of the projective face $K \cap \operatorname{im} Q^{*}$ corresponding to $Q$. Hence $\hat{w} \in K \cap \operatorname{im} P^{*}$, and so $P^{*} \hat{w}=\hat{w}$.

Using this and the definition of the bilinear form on $A_{f}$, we obtain

$$
\begin{aligned}
((I-P) u \mid P v) & =\lambda((I-P u \mid w) \\
& =\lambda\langle(I-P) u, \hat{w}\rangle=\lambda\left\langle u,(I-P)^{*} \hat{w}\right\rangle=0,
\end{aligned}
$$

which establishes $(6.8)$.

From (6.8) we conclude that

$$
(P u \mid v)=(P u \mid P v)=(u \mid P v)
$$

for every pair of atoms $u, v$. Now (6.6) follows by linearity, since by definition $A_{f}$ is the linear span of atoms. 
COROLLARY 6.5. If $P \in \mathcal{D}$ is finite or cofinite and $\varphi$ is the map defined in Lemma 6.4, then $\varphi(P a)=P^{*} \varphi(a)$ for all $a \in A_{f}$.

Proof. By (6.6) we have for all $a, b \in A_{f}$ :

$$
\langle b, \varphi(P a)\rangle=\langle b| P a)=\langle P b| a)=\langle P b, \varphi(a)\rangle=\left\langle b, P^{*} \varphi(a)\right\rangle
$$

By Corollary 6.3 it follows that $\varphi(P a)=P^{*} \varphi(a)$.

LемM 6.6. If a projective unit $u$ is finite (in the lattice $\mathcal{U}$ ), then it is a finite sum of orthogonal atoms, in particular it is in $A_{f}$.

Proof. Using the finite covering property, induction, and the formula

$$
u_{1} \vee \ldots \vee u_{n}=u_{1} \vee \ldots \vee u_{n-1}+\left(\left(u_{1} \vee \ldots \vee u_{n-1}\right) \vee u_{n}-u_{1} \vee \ldots \vee u_{n-1}\right),
$$

we conclude that $u$ is a finite sum of atoms. Since these atoms sum to $u \leqslant e$, they must be orthogonal [2; Prop. 4.4].

CoROLIARY 6.7. If $P \in \mathcal{D}$ is finite and $Q \lesssim P$, then $\left(Q+Q^{\prime}\right)^{*} \varphi(P e)=\varphi(P e)$.

Proof. By Corollary 6.5 and the compatibility of $P$ and $Q$ (cf. $[2 ;(5.4)])$

$$
\left(Q+Q^{\prime}\right)^{*} \varphi(P e)=\varphi\left(\left(Q+Q^{\prime}\right) P e\right)=\varphi(P e) .
$$

Proposition 6.8. Let $P \in \mathcal{D}$ be finite. Then every $\varrho \in \operatorname{im} P^{*}$ can be written as a finite linear combination $\varrho=\sum_{i=1}^{n} \lambda_{i} \varrho_{i}$ of orthogonal pure states $\varrho_{1}, \ldots, \varrho_{n} \in K \cap \operatorname{im} P^{*}$.

Proof. We proceed by induction on the (lattice theoretic) dimension of $P$.

If $\operatorname{dim} P=1$, then $P$ is minimal in $D$, and so im $P^{*}$ is one-dimensional with $K \cap \operatorname{im} P^{*}$ equal to a single extreme point [3; Prop. 1.13]. Hence the proposition holds in this case.

Let $n \geqslant 2$, and assume that the proposition holds for $\operatorname{dim} P<n$. Assume now that $\operatorname{dim} P=n$, and let $\varrho \in \operatorname{im} P^{*}$ be arbitrary. We write $v=P e$, and we note that $v \in A_{f}$ by Lemma 6.6. By the defining property $\varphi(u)=\hat{u}$ of the map $\varphi$, it maps orthogonal atoms to orthogonal pure states. Thus it follows from Lemma 6.6 that $\varphi(v)$ is a finite sum of orthogonal pure states. Hence if $\varrho=\lambda \varphi(u)$ for some $\lambda \in \mathbf{R}$, then we are done. Otherwise, there exists a $\lambda \in \mathbf{R}$ such that neither $\varrho \leqslant \lambda \varphi(v)$ nor $\varrho \geqslant \lambda \varphi(v)$. Hence, the element $\sigma \in V$ defined by $\sigma=\varrho-\lambda \varphi(v)$ is neither positive nor negative. By [3; Prop. 1.3], there exists $Q \in D$ with $Q \neq 0, Q \neq P, Q \lesssim P$, such that $\sigma^{+}=Q^{*} \sigma \geqslant 0, \sigma^{-}=-Q^{*} \sigma \geqslant 0$ and $\sigma=\sigma^{+}-\sigma^{-}$. Thus $\left(Q+Q^{\prime}\right)^{*} \sigma=\sigma$, and by Corollary 6.7 also $\left(Q+Q^{\prime}\right)^{*} \varphi(v)=\varphi(v)$; hence $\left(Q+Q^{\prime}\right)^{*} \varrho=\varrho$. Thus, we have a decomposition

$$
\varrho=\varrho_{1}+\varrho_{2}, \quad \varrho_{1}=Q^{*} \varrho, \quad \varrho_{2}=Q^{*} \varrho .
$$


Now $\varrho_{1} \in \operatorname{im} Q^{*}$ and $\varrho_{2} \in \operatorname{im} Q^{*} \cap \operatorname{im} P^{*}=\operatorname{im}\left(P Q^{\prime}\right)^{*}$. Since $Q \neq 0, Q \neq P$, then $\operatorname{dim} Q<\operatorname{dim} P$ and $\operatorname{dim}\left(P Q^{\prime}\right)<\operatorname{dim} P$. By the induction hypothesis, $\varrho_{1}$ is a finite linear combination of orthogonal pure states in $K \cap \operatorname{im} Q^{*}$ and $\varrho_{2}$ is a finite linear combination of orthogonal pure states in $K \cap \mathrm{im}\left(P Q^{\prime}\right)^{*}$. Since each pure state in $K \cap \mathrm{im} Q^{*}$ is orthogonal to each pure state in $K \cap \mathrm{im}\left(P Q^{\prime}\right)^{*}$, we can substitute the decompositions for $\varrho_{1}$ and $\varrho_{2}$ into $(6.9)$ and obtain a decomposition of the desired type for $\varrho$. This completes the induction.

COROLLARY 6.9. Every $a \in A_{f}$ can be written as a finite linear combination $a=\sum_{i=1}^{n} \lambda_{i} u_{i}$ of orthogonal atoms $u_{1}, \ldots, u_{n}$.

Proof. Let $a=\sum_{j=1}^{m} \gamma_{j} v_{j}$ where $v_{1}, \ldots, v_{m}$ are atoms. Let $v=v_{1} \vee \ldots \vee v_{m}$ and choose $P \in \mathcal{D}$ such that $P e=v$. Then $\varphi(a)=\sum_{j=1}^{m} \gamma_{j} \hat{v}_{j} \in \operatorname{im} P^{*}$ and $P$ is finite, so by Proposition 6.8 there exist orthogonal pure states $\varrho_{1}, \ldots, \varrho_{n} \in K \cap \operatorname{im} P^{*}$ and scalars $\lambda_{1}, \ldots, \lambda_{n}$ such that

$$
\sum_{j=1}^{m} \gamma_{j} \hat{v}_{j}=\sum_{i=1}^{n} \lambda_{i} \varrho_{i}
$$

Let $u_{1}, \ldots, u_{n}$ be the atoms in $\operatorname{im} P$ such that $\hat{u}_{i}=\varrho_{i}$ for $i=1, \ldots, n$. By (6.4) we have for every atom $w \in \operatorname{im} P$

$$
\left\langle a-\sum_{i=1}^{n} \lambda_{i} u_{i}, \hat{w}\right\rangle=\left\langle w, \sum_{j=1}^{m} \gamma_{j} \hat{v}_{j}-\sum_{i=1}^{n} \lambda_{i} \varrho_{i}\right\rangle=0
$$

By Proposition 6.8, $a-\sum_{i=1}^{n} \lambda_{i} u_{i}$ is annihilated by everything in im $P^{*}$; since im $P$ and $\operatorname{im} P^{*}$ are in separating duality $a=\sum_{i=1}^{n} \lambda_{i} u_{i}$, which proves the corollary.

Definition. For each atom $u=P e$ with $P \in \mathcal{D}$ and each $b \in A$ we write

$$
u * b=\frac{1}{2}\left(I+P-P^{\prime}\right) b
$$

The following lemma and proof are essentially those of $[7 ;$ Th. 5.1].

Lемма 6.10. Let $u, v$ be atoms in A. Then:

(i) $u * v=v * u$,

(ii) $u *(v * b)=v *(u * b)$ if $u \perp v$ and $b \in A$.

Proof. (i) Let $P, Q \in \mathcal{D}$ be such that $P e=u$ and $Q e=u \vee v$. On im $Q$ the quasicomplement of $P$ is just the restriction of $P^{\prime}$ [3; Prop. 1.10]; thus $u * v$ is the same whether calculated in $A$ or in $\operatorname{im} Q$. For simplicity of notation we will therefore assume $A=\operatorname{im} Q$, or equivalently $e=u \vee v$. Note that in this case $\operatorname{dim} e=2$, therefore $e-u$ is also an atom (Lemma 5.1). 
The space im $P$ is one-dimensional since $u$ is an atom [3; Prop. 1.13]. Hence $P v=\lambda u$ for some $\lambda \in \mathbf{R}$. Now by Lemma 6.4

$$
\lambda=\langle P v, \hat{u}\rangle=(P v \mid u)=(v \mid P u)=(v \mid u),
$$

hence

$$
P v=(v \mid u) u
$$

Since $e-u$ is also an atom, we can apply the same argument to $P^{\prime}$ and obtain

$$
P^{\prime} v=(v \mid e-u)(e-u)
$$

Now by the definition of $u * v$ :

$$
u * v=\frac{1}{2}[v+(v \mid u) u-(v \mid e-u)(e-u)]=\frac{1}{2}[u+v-(1-(v \mid u)) e]
$$

(Note that $(v \mid e)=\langle e \mid v\rangle=\langle e, \hat{v}\rangle=1$ ). The above expression is symmetric in $u$ and $v$, and so $u * v=v * u$.

(ii) Let $P, R \in \mathcal{D}$ be such that $P e=u$ and $R e=v$. Since $P$ and $R$ are orthogonal, then by [3; (5.5)] $P, R, P^{\prime}, R^{\prime}$ all commute; hence

$$
u *(v * b)=\frac{1}{4}\left(I+P-P^{\prime}\right)\left(I+R-R^{\prime}\right) b=\frac{1}{4}\left(I+R-R^{\prime}\right)\left(I+P-P^{\prime}\right) b=v *(u * b)
$$

This completes the proof.

Proposition 6.11. Let $A_{0}$ be the norm closure of the set of all elements $a+\lambda$ where $a \in A_{f}$ and $\lambda \in \mathbf{R}$. Then there exists a unique product $a \circ b$ on $A_{0}$ such that $A_{0}$ is a JB-algebra with identity $e$ and $u \circ v=u * v$ for all atoms $u, v$.

Proof. 1. We first define the product on $A_{f}$ by

$$
a \circ b=\sum_{i, j} \alpha_{i} \beta_{j} \quad u_{i} * v_{j}
$$

where $a=\sum_{i=1}^{n} \alpha_{i} u_{i}, b=\sum_{j=1}^{m} \beta_{j} v_{j}$ with $u_{1}, \ldots, u_{n}, v_{1}, \ldots, v_{m}$ atoms. It is easily seen from the definition (6.10) that the right hand side of (6.13) is independent of the particular representation of $b$. By Lemma 6.10 (i), it is also independent of the representation of $a$. Thus, $a \circ b$ is a well defined commutative bilinear product on $A_{f}$.

We will next verify that the fundamental Jordan identity

$$
a^{2} \circ(b \circ a)=\left(a^{2} \circ b\right) \circ a
$$

holds for $a, b \in A_{f}$. Note first that by the definition (6.10), $u * u=u$ and $u * v=0$ when $u \perp v$. Let $a, b$ be represented as before, but assume now that the atoms $u_{1}, \ldots, u_{n}$ are orthogonal (Corollary 6.9). Then 
while

$$
\left(a^{2} \circ b\right) \circ a=\sum_{i, j, k} \lambda_{i}^{2} \gamma_{j} \lambda_{k}\left(u_{i} \circ v_{j}\right) \circ u_{k}
$$

$$
a^{2} \circ(b \circ a)=\sum_{i, j, k} \lambda_{i}^{2} \gamma_{j} \lambda_{k i} u_{i} \circ\left(v_{j} \circ u_{k}\right)
$$

By Lemma 6.10, this proves (6.14). Hence $A_{f}$ is a Jordan algebra under the product (6.13).

2. If $e \in A_{f}$, then for every atom $u=P e$ with $P \in \nsupseteq$ we have

$$
u \circ e=\frac{1}{2}\left(I+P-P^{\prime}\right) e=P e=u,
$$

so $e$ is an identity for $A_{f}$.

If $e \notin A_{f}$, then we define for $a, b \in A_{f}$ and $\lambda, \mu \in \mathbf{R}$ :

$$
(a+\lambda e) \circ(b+\mu e)=a \circ b+\lambda b+\mu a+\lambda \mu e .
$$

It is straightforward to verify that with this product $A_{f}+\mathbf{R} e$ becomes a Jordan product with identity $e$.

3. To prove that $A_{f}+\mathrm{R} e$ satisfies the norm requirements $(2.1),(2.2),(2.3)$ in the definition of a $J B$-algebra, it suffices by [4; Th. 2.1] to show that

$$
-e \leqslant a \leqslant e \text { implies } 0 \leqslant a^{2} \leqslant e .
$$

(The proof of [4; Th. 2.1] is given for a norm complete algebra, but the completeness is not used in the proof.)

Let $a=\sum_{i=1}^{n} \lambda_{i} u_{i}+\gamma e$ where $u_{1}, \ldots, u_{n}$ are orthogonal atoms. Setting $q=e-\sum_{i=1}^{n} u_{i}$, we can rewrite the representation of $a$ in the form

$$
a=\sum_{i=1}^{n} \alpha_{i} u_{i}+\beta q .
$$

Note that $q^{2}=q$ and $q \circ u_{i}=0$ for all $i$. Since $u_{1}, \ldots, u_{n}, q$ are orthogonal projective units, one may apply suitable $P$-projections to (6.16) and obtain

$$
\|a\|=\max \left\{\left|\alpha_{1}\right|, \ldots,\left|\alpha_{n}\right|,|\beta|\right\} .
$$

Assume now that $-e \leqslant a \leqslant e$. Then $\left|\alpha_{i}\right| \leqslant 1$ for $i=1, \ldots, n$ and $|\beta| \leqslant 1$, and so

$$
\left\|\alpha^{2}\right\|=\left\|\sum_{i=1}^{n} \alpha_{i}^{2} u_{i}+\beta^{2} q\right\|=\max \left\{\left|\alpha_{i}^{2}\right|, \ldots,\left|\alpha_{n}^{2}\right|,\left|\beta^{2}\right|\right\} \leqslant 1,
$$

which proves (6.15).

4. By the inequality (2.1), i.e. $\|a \circ b\| \leqslant\|a\|\|b\|$, the product $a \circ b$ is norm continuous on $A_{f}+\mathbf{R} e$. Hence it can be extended by continuity to $A_{0}=\left(A_{f}+\mathbf{R} e\right)^{-}$, and $A_{0}$ is seen to be a $J B$-algebra for this product. 
Lemma 6.12. For each $a \in A_{0}$ and $\varrho \in V$ there exists a unique element ao $€ V$ such that

$$
\langle b, a \circ \varrho\rangle=\langle a \circ b, \varrho\rangle \text { for all } b \in A_{0} \text {. }
$$

Proof. Assume first that $a$ is an atom, say $a=P e$ with $P \in \mathcal{p}$. By the definition of the Jordan product on $A_{0}$, we have $a \circ b=\frac{1}{2}\left(I+P-P^{\prime}\right) b$, and hence

$$
\langle a \circ b, \varrho)=\left\langle b, \frac{1}{2}\left(I+P-P^{\prime}\right)^{*} \varrho\right\rangle
$$

for all $b \in A_{0}$. Thus, $a \circ \varrho=\frac{1}{2}\left(I+P-P^{\prime}\right)^{*} \varrho$ satisfies $(6.17)$ in this case. Note also that when $a=e$, then (6.17) is satisfied with $e \circ \varrho=\varrho$. By linearity, there exists $a \circ \varrho \in V$ satisfying (6.17) for every $a \in A_{f}+\mathbf{R} e$.

For an arbitrary $a \in A_{0}$ we choose a sequence $\left\{a_{n}\right\}$ from $A_{f}+\mathbf{R} e$ which converges in norm to $a$. By the norm-inequality (2.1) in the $J B$-algebra $A_{0}$ (and by Corollary 6.3), $\left\{a_{n} \circ \varrho\right\}$ will be a Cauchy sequence in $V$. By completeness of $V$, this sequence is convergent, and clearly the limit satisfies (6.17). Finally, by Corollary 6.3, the solution $a \circ \varrho$ to (6.17) is unique.

Proposition 6.13. A can be equipped with a Jordan product which makes it a JBW. algebra with predual $V$.

Proof. It follows from Corollary 6.3 that $A_{0}$ and $V$ are in separating order and norm duality. Hence $V$ can be embedded order isomorphically and isometrically into $A_{0}^{*}$. In the rest of this proof we shall assume that this embedding has been performed. Recall from $\S 2$ that $A_{0}^{* *}$ is a $J B W$-algebra with predual $A_{0}^{*}$. Note also that the identity element of $A_{0}^{* *}$ is (the canonical image of) the element $e \in A$. Since $A_{0}$ is $w^{*}$-dense in $A_{0}^{* *}$ and the Jordan (=Arens) product in $A_{0}^{* *}$ is separately $w^{*}$-continuous in each variable, the formula (6.17) will subsist for $b \in A_{0}^{* *}$. Thus, for $a \in A_{0}$ and $\varrho \in V$, then

$$
\langle b, a \circ \varrho\rangle=\langle a \circ b, \varrho\rangle \quad \text { for all } b \in A_{0}^{* *} \text {. }
$$

Now let $J$ be the polar of $V$ in $A_{0}^{* *}$. By (6.18) $a \circ J \subseteq J$ for $a \in A_{0}$. Since $J$ is $w^{*}$-closed, it follows that $a \circ J \subseteq J$ for all $a \in A_{0}^{* *}$. Hence $J$ is a $w^{*}$-closed Jordan ideal in $A_{0}^{* *}$. By [17; Lemma 2.1], there exists a central idempotent $c \in A_{0}^{* *}$ such that $J=U_{c}\left(A_{0}^{* *}\right)=\operatorname{im} U_{\mathrm{c}}$. Now it follows from [3; Prop. 1.10] that im $U_{e-c}$ is a $J B W$-algebra with predual im $U_{e-c}$. Since $V$ is complete, it is closed in $A_{0}^{*}$; by the bipolar theorem it is the polar of $J=\operatorname{im} U_{c}$, hence

$$
V=\operatorname{ker} U_{c}=\operatorname{im} U_{e-c}
$$

Thus $A=V^{*} \cong \operatorname{im} U_{e-c}$, and the proof is complete. 
Remark. It can actually be shown that the canonical mapping of $V$ in $A_{0}^{*}$ is onto. Hence the central idempotent $c$ of the proof above must be zero, and $A=V^{*} \cong A_{0}^{* *}$. However, we will not need this result in what follows, so we omit the proof.

With the above Proposition 6.13 we have completed our list of consequences of the assumptions $(6.1)-(6.4)$, and our discussion henceforth will only presuppose the standing hypotheses of this section $\left(A=V^{*}, V\right.$ is complete, and (2.5) holds). We are now ready for the main results of this section, which consist in necessary and sufficient conditions for $A$ to be an atomic $J B W$-algebra (and in particular a $J B W$-factor of type I). Note that this is an unambiguous statement, for if $A$ is a $J B W$-algebra for some product $a \circ b$, then $V$ is its (unique) predual, and by [3; Prop. 3.1] $A$ and $V$ are in spectral duality and the Jordan product is uniquely determined by

$$
a \circ b=\frac{1}{2}\left[(a+b)^{(2)}-a^{(2)}-b^{(2)}\right]
$$

where the exponent (2) indicates the squaring operation defined by spectral theory.

Propositron 6.14. A is a $J B W$-factor of type $I$ iff $A$ is a factor of type $I$ (as defined in $\S 2)$ and $K$ has the pure state properties.

Proof. The conditions are necessary since the normal state space of any $J B W$-algebra has the pure state properties $(\$ 4)$.

To prove the sufficiency, we assume that $A$ is a type I factor and that $K$ has the pure state properties. By Proposition 6.13 we only have to verify the properties (6.1)-(6.4). Of these, (6.3) and (6.4) are identical with the pure state properties (4.3) and (4.5), and (6.2) is satisfied since $\mathcal{F}$ has the covering property (cf. [3; Prop. 1.9]). Thus, only the atomicity of $\mathcal{I}$ has to be established.

In this connection we shall need the following general implication involving the projective unit $r(a)$ associated with a given $a \in A^{+}$(see [2; p. 31] for the definition):

$$
\text { If } P, Q \in D, a \in A^{+} \text {and } P a \in \operatorname{im} Q, \quad \text { then } P(r(a)) \in \operatorname{im} Q \text {. }
$$

To verify (6.20), observe that if $P a \in \operatorname{im} Q$, then $Q^{\prime} P a=0$, so a annihilates im $\left(Q^{\prime} P\right)^{*}=$ im $P^{*} Q^{* *}$. By [2; Prop. 4.7], $r(a)$ also annihilates $P^{*} Q^{*} \varrho$ for each $\varrho \in K$. Now it follows that $Q^{\prime} \operatorname{Pr}(a)=0$. Since $Q$ and $Q^{\prime}$ are quasicomplementary, we must have $P(r(a)) \in \operatorname{im} Q$.

We now turn to the proof of the atomicity of $\mathcal{F}$, which proceeds in three steps.

1. We will first verify that $e$ is equal to the l.u.b. $u_{0} \in \mathcal{U}$ of all atoms in $u$. (Note that $\mathcal{U}$ is a complete lattice by [3; Cor. 1.2], so this l.u.b. exists.)

We consider an arbitrary $P \in \mathcal{D}$, and we will show that $P$ is compatible with $u_{0}$. Let $\left[u_{0}\right]$ be the order-ideal of $A$ generated by $u_{0}$, so $\left[u_{0}\right]=\operatorname{im} Q$ where $u_{0}=Q e$ with $Q \in \mathcal{D}$ 
[2; Cor. 2.12]. If $u_{1}, \ldots, u_{n}$ is any finite set of atoms, then by Proposition 4.2 (ii), $P u_{1}, \ldots, P u_{n}$ are multiples of atoms; therefore

$$
P\left(u_{1}+\ldots+u_{n}\right) \in\left[u_{0}\right]
$$

By the definition of the map $a \mapsto r(a)$ (see [2; § 4], we have $r(a) \vee r(b)=r(a+b)$ for $a, b \in A^{+}$. Hence by the implication (6.20) above:

$$
P\left(u_{1} \vee \ldots \vee u_{n}\right)=P\left(r\left(u_{1}+\ldots+u_{n}\right)\right) \in\left[u_{0}\right] .
$$

By [2; Prop. 2.11], this shows that $P\left(u_{1} \vee \ldots \vee u_{n}\right) \leqslant u_{0}$. Clearly the family of all l.u.b.'s of finite sets of atoms is directed upwards with l.u.b. equal to $u_{0}$; by [2; Lemma 12.1] $u_{0}$ is also the weak limit of this directed family. We have just seen that $P$ maps each element of this family under $u_{0}$; by weak continuity of $P$ we conclude $P u_{0} \leqslant u_{0}$. By Proposition 5.1 of [2] this shows that $P$ is compatible with $u_{0}$.

Now we have proved that $u_{0}$ is compatible with any $P \in \mathcal{D}$, and so is by definition central. Since $A$ is a factor, either $u_{0}=0$ or $u_{0}=e$. Since $A$ is of type $I$, it contains at least one atom, so the first possibility is ruled out. Therefore $u_{0}=e$, as desired.

2. Next we will show that for an arbitrary non-zero element $u \in \mathcal{U}$ where $u=P e$ with $P \in \mathcal{D}$, there exists at least one atom $w$ such that $w \leqslant u$. If $v$ is any atom, then by Proposition 4.2, $P v$ is either a non-zero multiple of an atom or $P v=0$. In the former case $w=\|P v\|^{-1} P v=r(P v)$ is an atom satisfying $P v \leqslant P e=u$, so our claim is verified. We will show that the latter case can not prevail for all atoms $v \in \mathcal{U}$. In fact, if $P v=0$ where $v=Q e$ with $Q \in \mathcal{D}$, then $P Q e=0$, which implies $P \perp Q$, and so $u \perp v$ (cf. [2; p. 28]). Thus, if $P v=0$ for all atoms $v$, then $v \leqslant u^{\prime}=e-u$ for all atoms $v$; since $e$ has been shown to be the l.u.b. of atoms, this gives $e \leqslant e-u$, i.e. $u=0$, contrary to assumption.

3. Again we consider an arbitrary non-zero element $u \in \mathcal{U}$. By Zorn's lemma there exists a maximal orthogonal family $\left\{u_{\alpha}\right\}$ of atoms under $u$. Let $v \in \mathcal{U}$ be the 1.u.b. of $\left\{u_{\alpha}\right\}$. Now we must have $u=v$, for otherwise $u-v$ would contain an atom which would be orthogonal to all $u_{\alpha}$, contrary to the maximality of $\left\{u_{\alpha}\right\}$. This completes the proof.

Proposition 6.15. $A$ is an atomic $J B W$-algebra iff $K$ has the Hilbert ball property and is the $\sigma$-convex hull of its extreme points.

Proof. The normal state space of any $J B W$-algebra has the Hilbert ball property $(\S 3)$, and by $\S 5$ the normal state space of an atomic $J B W$-algebra is the $\sigma$-convex hull of its extreme points. Hence the conditions are necessary.

To prove sufficiency, we assume that $K$ has the Hilbert ball property and is the $\sigma$-convex hull of its extreme points. Again we only have to verify (6.1)-(6.4). Of these, (6.3) 
is explicitly required in the definition of the Hilbert ball property, and (6.4) follows from the Hilbert ball property by Proposition 4.3. Hence we have left the finite covering property and the atomicity of $₹$.

We first establish the atomicity. Let $G \in \mathcal{F}$ and let $\varrho \in G$. By assumption $\varrho=\sum_{i=1}^{\infty} \varrho_{i}$ where $\sum_{i=1}^{\infty} \lambda_{i}=1$ and $\lambda_{i} \geqslant 0, \varrho_{i} \in \partial_{e} K$ for $i=1,2, \ldots$. Choosing $k$ such that $\lambda_{k} \neq 0$, we have

$$
\varrho=\lambda_{k} \varrho_{k}+\left(1-\lambda_{k}\right)\left[\left(1-\lambda_{k}\right)^{-1} \sum_{i \neq k} \lambda_{i} \varrho_{i}\right]
$$

Since $G$ is a face of $K$, then $\varrho_{k} \in G$. Thus, $G$ contains the minimal projective face $\left\{\varrho_{k}\right\}$, and the atomicity is verified.

The finite covering property is most easily established for the lattice $u$. Then we must show that if $u \in \mathcal{U}$ is an atom and $w \in \mathcal{U}$ is any finite element, then

$$
u \vee w-w \text { is an atom or zero. }
$$

We will prove (6.21) by induction on the (lattice) dimension of $w$. If $\operatorname{dim} w=1$, then $w$ is an atom and (6.21) follows from the Hilbert ball property (since the only proper faces of a Hilbert ball are the extreme points). Let $\operatorname{dim} w=n \geqslant 2$ and assume that (6.21) holds for all smaller dimensions of $n$. Choose atoms $w_{1}, \ldots, w_{n}$ such that $w=w_{1} \vee \ldots \vee w_{n}$, and note that the element $v=w_{1} \vee \ldots \vee w_{n-1}$ satisfies $\operatorname{dim} v<n$ and $v \neq w(\operatorname{since} \operatorname{dim} w=n)$. Now

$$
0 \leqslant u \vee w-w<u \vee w-v
$$

By the Hilbert ball property, we will have established $(6.21)$ if we show $\operatorname{dim}(u \vee w-v) \leqslant 2$.

To prove this, we first observe that the map $x \mapsto x-v$ is a lattice isomorphism of the lattice interval $[v, e]$ onto $[0, e-v]$; thus since $v \leqslant w$ :

$$
u \vee w-v=(u \vee v) \vee w-v=(w-v) \vee[(u \vee v)-v]
$$

By induction hypothesis both $w-v$ and $u \vee v-v$ are atoms or zero; hence the dimension of their join is at most 2 , which finishes the proof.

Note that by the definitions, the atomic $J B W$-algebra $A$ of Proposition 6.15 is a $J B W$-factor of type I precisely if it is a type I factor in the sense defined in $\S 2$.

In the following theorem the main results of this section are restated in a more geometric form. Here the starting point is a convex set $C$ (the normal state space) which is supposed to be embedded as the base of a complete base-norm space; as usual the term "projective face" refers to the duality with the order-unit space of all bounded affine functions on $C$. 
Theовем 6.16. Let $C$ be the base of a complete base-norm space. Then $C$ is affinely isomorphic to the normal state space of a $J B W$-factor of type $I$ iff $C$ satisfies (i), (ii) and either (iii) or (iii)' below:

(i) C contains no proper split faces

(ii) Every norm-exposed face of $C$ is projective

(iii) $C$ contains at least one extreme point and $C$ has the pure state properties

(iii)' $C$ is the $\sigma$-convex hull of its extreme points and $C$ has the Hilbert ball property.

Proof. Assume first that $C$ is the normal state space of a type I $J B W$-factor $A$. Then $A$ can be identified with the space of all bounded affine functions on $C$ (cf. [3; Th. 3.1]); there can be no proper split faces in $C$ since $A$ is a factor [2; Prop. 10.2], and $\partial_{e} C \neq \varnothing$ since $A$ is of type $\mathrm{I}$. By the results of $\S 3,4, C$ has the Hilbert ball property and the pure state properties. It was shown in $\S 5$ that the $\sigma$-convex hull of $\partial_{e} C$ is a split face of $C$; since it is non-empty, it must be equal to $C$. With this we have proved that the conditions are necessary.

The sufficiency of (i), (ii) and (iii) follows from Proposition 6.14; the sufficiency of (i), (ii) and (iii)' follows from Proposition 6.15.

\section{\$ 7. The main theorem}

In this section $K$ will be a compact convex set. Without loss of generality we assume that $K$ is embedded as the base of the base-norm space $V=A(K)^{*}$ where $A(K)$ is the space of all continuous affine functions on $K[1 ; \mathrm{Ch}$. II, $\S 2]$. As before, we denote by $A \cong V^{*}$ the space of all bounded affine functions on $K$, and we recall that $(A, e)$ is an order-unit space in separating order and norm duality with $(V, K)$. In the sequel all notions from spectral theory, like "projective face" "projective unit" and "orthogonality" ( $a \perp b$ for $a, b \in A^{+}$), will refer to this duality.

Recall from $[3 ; \S 1]$ that if every norm exposed face of $K$ is projective, then for every $\varrho \in \partial_{e} K$ there exists a smallest split face $F_{\varrho}$ containing $\varrho$; the corresponding central projective unit is called the central support of $\varrho$ and is denoted $e(\varrho)$. Also we will use the notation $A_{\varrho}=\operatorname{im} P_{\varrho}$ where $P_{\varrho}$ is the $P$-projection corresponding to $F_{\varrho}$.

The next result shows that there are many "type I factor representations"; this will allow us to "globalize" the "local" results of $\S 6$.

Lемма 7.1. If every norm exposed face of $K$ is projective, then $A_{\varrho}$ is a type $I$ factor for every norm-exposed $\varrho \in \partial_{e} K$. 
Proof. Suppose for contradiction that $A_{e}$ is not a factor. Then by [3; Cor. 1.11] there exists a central $P$-projection $Q \in \mathcal{D}$ such that $Q \precsim P_{\varrho}, Q \neq 0, Q \neq P_{\varrho^{\circ}}$. Since $Q$ is central, $Q+Q^{\prime}=I$. Hence $\left(Q+Q^{\prime}\right)^{*} \varrho=\varrho$. Since $\varrho$ is extreme, $Q^{*} \varrho$ and $Q^{\prime *} \varrho$ must both be multiples of $\varrho$. Then necessarily $Q^{*} \varrho=0$ or $Q^{\prime *} \varrho=0$; otherwise $Q^{*} \varrho$ would be a non-zero multiple of $Q^{\prime *} \varrho$ which is impossible.

Now let $c_{1}=Q e$ and $c_{2}=c(\varrho)-Q e$. By hypothesis $0<c_{1}<c(\varrho)$ and $c_{1}, c_{2}$ are central projective units such that $c_{1}+c_{2}=c(\varrho)$. If $Q^{*} \varrho=0$, then $\left\langle c_{1}, \varrho\right\rangle=\langle Q e, \varrho\rangle=0$, so $\left\langle c_{2}, \varrho\right\rangle=$ $\left\langle c(\varrho)-c_{1}, \varrho\right\rangle=1$. If $Q^{\prime *} \varrho=0$, then we similarly get $\left\langle c_{1}, \varrho\right\rangle=1$. In either case we have a contradiction with the minimality requirement defining $c(\varrho)$.

THновем 7.2. A compact convex set $K$ is affinely homeomorphic to the state space of a $J B$-algebra (with $w^{*}$-topology) iff $K$ satisfies the following requirements:

(i) $K$ has the Hilbert ball property

(ii) The $\sigma$-convex hull of the extreme points of $K$ is a split face of $K$

(iii) Every norm exposed face of $K$ is projective

(iv) Every $a \in A(K)$ admits a decomposition $a=a^{+}-a^{-}$where $a^{+}, a^{-} \in A(K)^{+}$and $a^{+} \perp a^{-}$.

Proof. If $K$ is affinely homeomorphic to the state space of a $J B$-algebra, then (i) holds by Corollary 3.12 and (ii) holds by Corollary 5.8 ; moreover, $K$ is strongly spectral by [3; Cor. 3.2] and so it satisfies (iii) and (iv). Hence the conditions are necessary.

To prove sufficiency, we assume that (i)-(iv) are satisfied. By [3; Prop. 1.10] for each $\varrho \in \partial_{e} K$ the split face $F_{\varrho}$ will also satisfy (i), (ii), (iii) and $A_{\varrho}$ can be identified with the space of all bounded affine functions on $F_{\varrho}$. Thus by Proposition 6.15 and Lemma 7.1, each $F_{Q}$ is the normal state space of the $J B W$-factor $A_{Q}$.

For each $\varrho \in \partial_{e} K$ let $\varphi_{\ell}$ denote the map which sends $a \in A(K)$ to $a \mid F_{\ell} \in A_{\varrho}$. Also define $B=\oplus \sum_{\varrho \in \partial_{e} K} A_{\varrho}$, and note that $B$ is a $J B$-algebra with pointwise operations and the supremum norm. Note also that by the Krein-Milman theorem the map $\varphi: a \mapsto \sum_{\ell \in \theta_{e} K} \varphi_{\ell}(a)$ is an isometric order-isomorphism of $A(K)$ into $B$.

Now let $a \in A(K)$, and let $a=a^{+}-a^{-}$be a decomposition of the type described in (iv). By [3; Prop. 1.10], $\varphi_{\varrho}\left(a^{+}\right)$and $\varphi_{\varrho}\left(a^{-}\right)$will be orthogonal in $A_{\varrho}$ for given $\varrho \in \partial_{e} K$. By [3; Th. 3.1] the $J B W$-algebra $A_{\varrho}$ is in spectral duality with its predual; hence the decomposition of $\varphi_{\varrho}(a)$ as a difference of two orthogonal elements of $A_{\varrho}$ is unique [3; Th. 2.2]. It follows that $\varphi_{Q}\left(a^{+}\right)=\varphi_{Q}(a)^{+}$.

By [3; Th. 3.1] the squaring maps given by the Jordan product in $A_{Q}$ and by the spectral duality of $A_{\varrho}$ with its predual, will coincide. Thus, by the Stone-Weierstrass theorem the element $\varphi_{\ell}(a)^{+}$defined by spectral theory in $A_{\ell}$ coincides with the positive part of $\varphi_{e}(a)$ in the $J B$-algebra sense. Working in the $J B$-algebra $B$, we now obtain 


$$
\varphi(a)^{+}=\sum_{e^{\epsilon} \theta_{e^{K}}} \varphi_{Q}(a)^{+}=\sum_{e \in \hat{\theta}_{e}} \varphi_{Q}\left(a^{+}\right)=\varphi\left(a^{+}\right) .
$$

Thus, $\varphi(A(K)))$ is a norm closed subspace of $B$ which contains the identity and is closed under the map $a \mapsto a^{+}$. Applying the lattice version of the Stone-Weierstrass theorem, we find that $\varphi(A(K))$ is closed under squaring. Hence $\varphi(A(K))$ is a $J B$-subalgebra of $B$.

Since $\varphi$ is an isometric order-isomorphism of $A(K)$ onto the $J B$-algebra $\varphi(A(K))$ such that $\varphi(e)=e$, then $\varphi^{*}$ is an affine homeomorphism of the state space of $\varphi(A(K))$ onto the state space of $A(K)$, i.e. onto $K$ itself. This completes the proof.

The first one of the corollaries below is actually an alternative, but somewhat less geometric, characterization of state spaces of $J B$-algebras.

CoROLLARY 7.3. A compact convex set $K$ is the state space of a JB-algebra iff it satisfies (iii), (iv) and in addition:

(v) $K$ has the pure state properties.

Proof. The proof is similar to that of Theorem 7.2, one only has to use Proposition 6.14 instead of Proposition 6.15 to prove that $A_{\varrho}$ is a $J B W$-factor for each $\varrho \in K$.

CoROLLARY 7.4. A compact convex set $K$ is the state space of a JB-algebra iff it is strongly spectral and satisfies (i) and (ii).

Proof. Strong spectrality is necessary by [3; Cor. 3.2], and it is sufficient since it implies (iii) and (iv) (cf. [3; Th. 2.2]).

Clearly one may replace (i) and (ii) by (v) also in Corollary 7.4. Note also that for a general compact convex set $K$, the requirement that $K$ be strongly spectral is strictly stronger than (iii) and (iv) together (cf. the example in [3; Prop. 2.5]).

Corollary 7.5. A finite dimensional compact convex set $K$ is the state space of a $J B$-algebra iff it satisfies (i) and (iii).

Proof. In the finite dimensional case (ii) holds automatically, and by [2; Th, 7.11] (iv) follows from (iii).

We close this paper by a characterization of the state spaces of Jordan operator algebras. (See $\S 2$ for the definition.) In [4; Lemma 9.4] the Jordan operator algebras were characterized among all $J B$-algebras by means of a certain algebraic identity. Our present criterion may be considered as a geometric counterpart to this identity, and it will be stated in terms of the Hilbert balls $B(\varrho, \sigma)=$ face $(\{\varrho, \sigma\})$ determined by pairs $\varrho, \sigma$ of extreme points of the state space. 
Proposition 7.6. Let $B$ be a JB-algebra with state space $K$. Then we have the following two mutually exlcusive alternatives:

(i) $B$ is isomorphic to a Jordan operator algebra

(ii) There exists a pair $\varrho, \sigma$ of extreme points of $K$ such that $\operatorname{dim} B(\varrho, \sigma)=9$ and $B(\varrho, \sigma)$ is not a split face of $K$.

Proof. Suppose that $\varrho, \sigma \in \partial_{e} K, \operatorname{dim} B(\varrho, \sigma)=9$, and $B(\varrho, \sigma)$ is not a split face. Let $u$ and $v$ be the atoms in $A_{\varrho}$ corresponding to $\varrho$ and $\sigma$. Note that since $B(\varrho, \sigma)$ is not a split face of $K$, then $B(\varrho, \sigma)$ is a proper face of the split face $F_{\varrho}$, and so $u \vee v \neq e(\varrho)$ (the identity of $\left.A_{\varrho}\right)$. Since $\operatorname{dim}(u \vee v)=2$, we can choose orthogonal atoms $p_{1}, p_{2}, p_{3}$ in $A$ such that $p_{1}+p_{2}=u \vee v$

Let $M=\left\{\left(p_{1}+p_{2}+p_{3}\right) A_{Q}\left(p_{1}+p_{2}+p_{3}\right)\right\}$. Then by Lemma $3.8, M$ is a type $I J B W$ factor. By Lemma 5.1, every set of orthogonal non-zero idempotents in $M$ has cardinality at most three, so $M$ is an $I_{3}$-factor.

Now by [4; Prop. 8.3], $M$ is isomorphic to the Jordan algebra of self-adjoint $3 \times 3$ matrices over the reals, complexes, quaternions, or the Cayley numbers. The same result holds with $2 \times 2$-matrices for

$$
\left\{\left(p_{1}+p_{2}\right) M\left(p_{1}+p_{2}\right)\right\}=\{(u \vee v) M(u \vee v)\}
$$

The state space of this $J B$-algebra is just $B(\varrho, \sigma)$. Counting dimensions, we conclude that $M$ is isomorphic to the exceptional algebra $M_{3}^{8}$ of all self-adjoint $3 \times 3$-matrices over the Cayley numbers.

Now if $B$ were isomorphic to a Jordan operator algebra, then $B$ and $B^{* *}$ would satisfy the crucial algebraic identity of [4; Lemma 9.4]. Since this identity is not satisfied in $M_{3}^{8} \cong M \subseteq B^{* *}$, this gives a contradiction. Hence $B$ can not be a Jordan operator algebra in this case.

Conversely, if $B$ is a $J B$-algebra non-isomorphic to a Jordan operator algebra, then by [4; proof of Lemma 9.4] some $A_{\varrho}$ will be isomorphic to $M_{3}^{8}$. Now for $\sigma \in \partial_{e} F_{\varrho}, \sigma \neq \varrho$ we shall have $\operatorname{dim} B(\varrho, \sigma)=9$ and $B(\varrho, \sigma)$ is a non-split face of $F_{\varrho}$ and hence of $K$. This completes the proof.

Acknowledgements. The second author gratefully acknowledges support from a research grant from Wellesley College during a year spent on leave at the University of California, Berkeley. He would also like to express his gratitude to the Mathematics Department in Berkeley and especially to Professors William Bade and Marc Rieffel for their hospitality during the time he spent in Berkeley. 


\section{References}

[1]. ALFSEn, E. M., Compact convex sets and boundary integrals. Ergebnisse der Math. 57, Springer Verlag, Berlin 1971.

[2]. Alfsen, E. M. \& Shultz, F. W., Non-commutative spectral theory for affine function spaces on convex sets. Mem. Amer. Math. Soc., 172, Providence R.I., 1976.

[3]. - On non-commutative spectral theory and Jordan algebras. Proc. London Math. Soc. To appear.

[4]. Aufsen, E. M., Shultz, F. W. \& Støryer, E., A Gelfand-Neumark theorem for Jordan algebras. Advances in Math. To appear.

[5]. Brzknoff, G., Lattice theory. Amer. Math. Soc. Colloq. Publ., Vol. 25, 3rd. edition, Providence, R.I. 1967.

[6]. BraUn, H. \& Koecher, M., Jordan Algebren. Springer Verlag, Berlin 1966.

[7]. Gunsor, J., On the algebraic structure of quantum mechanies. Comm. Math. Phys., 6 (1967), 262-285.

[8]. JACOBSON, N., Structure and representations of Jordan algebras. Amer. Math. Soc. Colloq. Publ. Vol. 39, Providence R.I. 1968.

[9]. Jordan, P., von Neumann, J.\& Wigner, E., On an algebraic generalization of the quantum mechanical formalism. Ann. of Math., 35 (1934), 29-64.

[10]. Maclaren, M. D., Atomic orthocomplemented lattices. Pacific J. Math., 14 (1964), $597-612$.

[11]. Maeda, F. \& MaEda, S., Theory of symmetric lattices. Grundlehren der math. Wissensch., No. 173, Springer Verlag, Berlin 1970.

[12]. Mrelnik, B., Theory of filters. Comm. Math. Phys., 15 (1969), 1-46.

[13]. von NEUMANN, J., On an algebraic generalization of the quantum mechanical formalism, Part I. Mat. Sb., 1 (1936), 415-484.

[14]. Poor, J. C. T., Semimodularity and the logic of quantum mechanics. Comm. Math. Phys., 9 (1968), 218-228.

[15]. Schremer. E., Modular pairs in orthomodular lattices. Pacific J. Math., 91 (1966), 519-528.

[16]. Segad, I. E., Postulates for general quantum mechanics. Ann. of Math., 48 (1947), 930-948.

[17]. SHuLtz, F. W., On normed Jordan algebras which are Banach dual spaces. J. Functional Analysis. To appear.

[18]. Topprnc, D., Jordan algebras of self-adjoint operators. Mem. Amer. Math. Soc., 53, Providence R.I., 1965.

[19]. - An isomorphism invariant for spin factors. J. Math. Mech., 15 (1966), 1055-1064.

[20]. - Asymptoticity and semimodularity in projection lattices. Pacific J. Math., 20 (1967), 317-325.

Received January 3, 1977 